\title{
Nonlinear vibration analysis of rotating beams undergoing parametric instability: Lagging-Axial motion
}

\author{
Hadi Arvin* \\ Faculty of Engineering, Shahrekord University, Shahrekord, 115, IRAN \\ Andrea Arena ${ }^{1, *}$, Walter Lacarbonara ${ }^{2, *}$ \\ Department of Structural and Geotechnical Engineering, Sapienza University of Rome, Rome, 00184, ITALY
}

\begin{abstract}
The nonlinear free vibration and principal parametric resonance of rotating beams are investigated taking into account the lagging-axial coupling motion due to Coriolis force. This work tackles analytically the problem of parametric resonances induced by periodic modulation of the angular speed. The nonlinear equations of motion are obtained via a direct Lagrangian formulation. The method of multiple scales is employed to perform a perturbation analysis of the nondimensional equations of motion to deliver the effective nonlinearity of the lagging and axial modes and the critical conditions for the onset of parametric resonances. A comprehensive study on the effect of the rotational speed and the damping ratio on the modes nonlinearity and on the instability regions is presented. Comparisons in terms of effective nonlinearity coefficient and principal parametric resonance response were carried out so as to illustrate the importance of the exact geometrical formulation against ad hoc beam theories such as the Euler-Bernoulli beam model.
\end{abstract}

Keywords: Rotating beams, nonlinear free vibrations, Coriolis force, method of multiple scales, effective nonlinearity coefficient, parametric resonance.

\section{Introduction}

The wide use of rotating blades in aerospace industries, wind, water and gas turbines requires a very accurate design involving, necessarily, sophisticated mechanical modeling. Rotating blades can suffer different instabilities due to their flexibility and light damping. When a blade is subjected to certain critical angular speeds, several autoparametric resonances can be excited involving various modes, among which axial, flapping and lagging modes. Besides energy transfers between modes giving rise to multimode vibrations, single-mode parametric resonances can be excited when the angular speed has a slight periodic modulation such that the modulation frequency is close to twice

\footnotetext{
*Corresponding author: E-mail address: hadi.arvin@sku.ac.ir, Tel.: +98-32-324438, Fax: +98-32-324438

${ }^{1}$ E-mail address: andrea.arena@uniroma1.it, Tel.: +39-06-44585885

${ }^{2}$ E-mail address: walter.lacarbonara@uniroma1.it, Tel.: +39-06-44585111
} 
the frequency of the parametrically driven mode. This can happen due to rotor unbalances or as a consequence of prescribed ramps of the angular speed. This work addresses this kind of parametric resonance by discussing the instability regions for various modes obtained in semi-closed form.

More complex instability scenarios can involve multi-mode parametric resonances of sum or difference type whereby the mode frequencies are combined with the angular speed modulation frequency to generate resonant frequencies. These types of resonances are not covered in the present work. In addition, nonlinear free vibrations of rotating beams, whose kinematics are described by an exact geometrical formulation, are examined. A deep investigation of the effect of the rotating speed and the damping ratio on the nonlinear interactions between the beam lagging and axial normal modes and the parametric resonance excitations are unfolded both as critical and post-critical conditions. The importance of accounting for large amplitude deformations in slender structural elements is largely documented in the literature.

Among several studies performed in the past, a novel geometrically exact nonlinear model of highly deformable wings coupled with aerodynamic models, including nonlinearities associated with the presence of aerodynamic stall and flow separation, was proposed by Arena et al. [1] to study the aeroelastic behavior of HALE wings for an improved understanding of the nonlinear phenomena occurring when the unsteady aerodynamic effects and dynamic stall contribute more significantly to the wing dynamic behavior.

Hodges and Dowell [2] developed a formulation for rotating asymmetric, slender blades with spanwise variable pretwist angle and a small precone angle. The authors introduced important nonlinear terms such as the flapping-lagging inertial coupling terms and the bending-torsional coupling terms which they may destabilize the hingeless rotor. The Hamilton 's principle was employed by Crespo da Silva and Hodges [3] to obtain the nonlinear equations of motion of a rotating blade with a precone angle and a variable pitch angle. These equations were adopted in a successive work [4] to investigate the equilibrium and stability of a uniform cantilever untwisted rotor blade in hover. The authors inferred that the most significant cubic nonlinear terms playing a crucial role in the instability of the blade are the structural geometric nonlinearities in the torsional equation of motion. By considering the warping displacements and the Rodrigues angles, Hodges developed in [5] a nonlinear intrinsic formulation for the dynamic analysis of rotating pre-curved and pre-twisted anisotropic blades. The governing equations of motion were obtained via a mixed approach, resorting to Newtonian and variational procedures. In [6], Hodges clarified the special influences of the boundary conditions and the effects of the centrifugal forces on the eigenvalue problem of rotating Timoshenko beams. Pesheck et al. [7] employed the invariant manifolds to construct the nonlinear normal modes of rotating beams. The equations of motion were derived by using the Hamilton's principle based on the von Karman strain-displacement relationship. The authors calculated the nonlinear normal modes to obtain the reduced-order models for rotating beams. 
By resorting to Hamilton 's principle, Avramov et al. [8] obtained the equations of motion for a slender rotating beam with variable cross section. The eccentricity of the elastic center with respect to the center of mass was considered and the interaction between the flexural and torsional modes was examined. The authors employed the method of multiple scales (MMS) to study the type of nonlinearity in rotating blades. Saravia et al. [9] implemented the principle of virtual work to derive the governing equations of thin-walled composite rotating blades. The warping of the cross section and the eccentricity between the elastic and the center of mass were considered and the parametric resonance of the blade was investigated by using the finite element method. The authors concluded that by increasing the blade rotating speed the instability regions shift to the right. Turhan and Bulut [10] developed a formulation for inward and outward oriented rotating beams regarding the geometric and inertial nonlinearities up to the cubic order. They applied the Lindstedt-Poincaré method on the discretized equations of motion obtained by Galerkin projection to define the frequency response curves. The authors showed that the type of nonlinearity can suffer changes by variations of the rotating speed. Valverde and Garcia-Vallejo [11] implemented an absolute nodal coordinate formulation (ANCF) as well as a geometrically exact nonlinear formulation based on the Cosserat theory of rods to model the rotating beams. The found instability was related to a nontrivial configuration at a certain speed. By comparing the results of the ANCF with those obtained via exact formulation, the authors showed that the instability is artificially related to the ANCF. Qin and $\mathrm{Li}$ [12] studied the natural frequencies of rotating composite beams in hygrothermal environment. The outcomes reveal the unappreciable influence of the temperature change and moisture concentration on the natural frequencies. Sabater and Rhoads [13] examined the parametric system identification of a viscoelastic microbeam with large amplitude vibrations by curve fitting the experimental data using the approximate solution obtained by the averaging method. The outcomes clarify that the approximate solution can give rise to some issues in the determination of the precise parameters. Tresser and Bucher [14] developed a new formulation to defeat the inability of measuring deformation for parametrically excited low-speed spinning shafts subjected to unbalance forces. The proposed method increases the sensitivity to the balancing process for such unbalanced forces without needing to rotate the shaft in the high-speed region.

A geometrically exact formulation of rotating blades, based on the Cosserat theory of rods, was developed by Lacarbonara et al. [15]. The formulation accounted for the pre-twist angle and the eccentricity between the mass and elastic centers. The internal constraint of unshearability was introduced in the mechanical formulation and the exact expressions of the flapping-torsional and lagging-axial equations of motion were linearized about the prestressed configuration by employing the Taylor expansion. The authors conducted parametric studies to investigate the influence of the rotating speed and the Coriolis forces on the flapping, lagging, torsional and axial natural frequencies. Arvin et al. [16] derived the third order flapping and axial equations of motion for rotating isotropic 
blades by applying the Taylor expansion on the exact formulation developed in [15]. The direct MMS was applied to the third-order equations of motion so as to investigate the effects of the rotating speed on the softening or hardening behaviour of isotropic rotating beams in the flapping modes. Arvin and Lacarbonara [17] extended their previous work [15] to composite rotating blades. They derived the exact constitutive relations for the composite rotating blades employing the 3dimensional continuum theory. The direct MMS was employed to study the flapping-axial motion of the rotating composite blades. Arvin and Bakhtiari-Nejad [18] employed the Hamilton 's principle to derive the governing equations of a rotating composite Timoshenko beam. They applied the direct MMS to calculate the nonlinear normal modes of the rotating composite Timoshenko beams in the absence of the internal resonances. They investigated the effects of the rotation speed and the number of layers on the natural frequencies and the flapping backbone curves.

Nonlinear vibrations of rotating cylindrical shells undergoing thermo-mechanical loading were investigated by Liu et al. [19]. The outcomes reveal the important effect of the parametric excitation rather than the external excitation in the nonlinear dynamic response of the structure. Arvin [20] employed the first order direct MMS versus the Runge-Kutta method to study the instability region of a rotating isotropic beam which undergoes principal parametric resonance due to the rotating speed variation. The equations of motion were based on the von Karman strain-displacement relation. The author examined the damping coefficient and the mode number influences on the parametric resonance instability regions. Some differences between the instability regions predicted by the two mentioned methods were observed. To improve the understanding of the observed differences between the numerical analysis and the MMS, Arvin et al. [21] applied the second order MMS vs. the differential quadrature method to the governing equations of the rotating beams. The authors further demonstrated that the instability region computed via second order MMS was very close to that obtained via numerical analysis. Heidari and Arvin [22] studied the nonlinear free vibrations of rotating Timoshenko beams reinforced by carbon nanotubes (CNT) employing the direct MMS. The outcomes revealed that the type of nonlinearity depends on the type of CNT distribution profile and the rotation speed for thin beams while for thick beams just the CNT distribution profile has the decisive role. Effects of the CNT weight fraction onto the flutter and post-flutter condition of nanocomposite panels were recently studied in [23] by means of the multiple scales perturbation analysis which was carried out to characterize the flutter boundaries and the type of Hopf bifurcation at flutter.

To the best of the authors' knowledge, the nonlinear response of rotating beams subject to parametric resonances was not investigated in the literature. Hence, in this paper nonlinear vibrations of the lagging-axial motion of symmetric rotating beams are deeply examined and the influence of the Coriolis forces on the onset of the parametric resonance is investigated. For symmetric beams, the flapping-torsional motions are coupled to each other due to the Coriolis forces which also cause 
the coupling in the lagging-axial motions. Starting from the nonlinear mechanical model developed by these authors in [15], the direct MMS is applied to the coupled equations of motion to characterize the effective nonlinearity coefficient and to describe the stability regions of the parametric resonance of the lagging-axial motion. In particular, case-studies are proposed to show the effects of the angular speed and the damping ratio on the aforementioned nonlinear features of the parametric response of the lowest three lagging modes and the first and second axial modes, respectively. Finally, comparisons in terms of effective nonlinearity coefficient and principal parametric resonance response were carried out so as to illustrate the importance of the exact geometrical formulation against ad hoc beam theories such as the Euler-Bernoulli beam model.

\section{Mechanical model of rotating beams}

The parametric model proposed in this work to study the nonlinear dynamic response of rotating beams is based on a geometrically exact semi-intrinsic theory which yields the equations of motion obtained within the context of an Updated Lagrangian Formulation (ULF) and fully derived in a recent work of the present authors [15]. In particular, to describe the motion of the rotating beam, two configurations are considered, namely, the prestressed equilibrium configuration, induced by the centrifugal forces, and the current dynamic configuration $\mathcal{B}$. The latter, together with a close-up view of the beam cross section, is schematically depicted in Fig. 1.

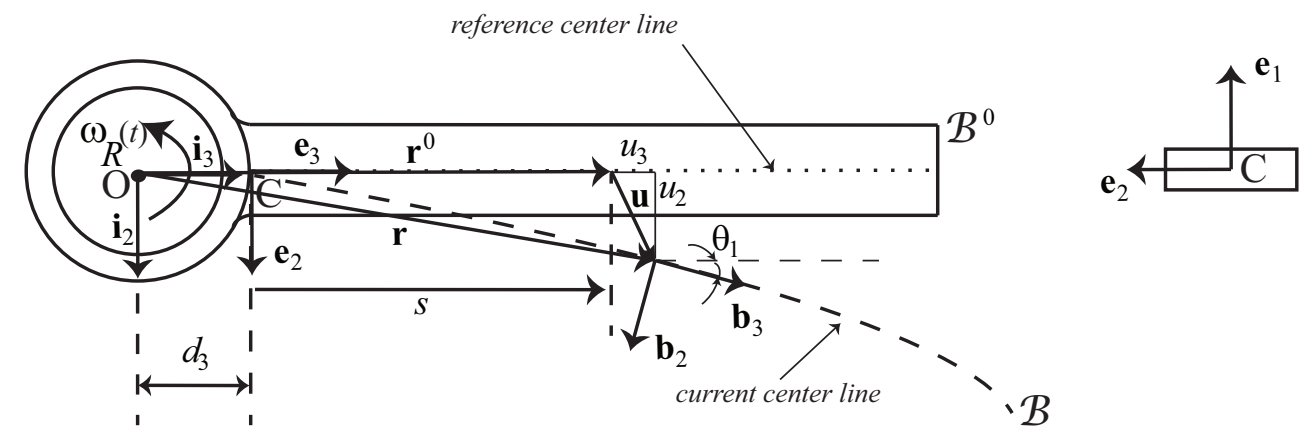

Figure 1: Schematic of the top-view of the beam in the stress-free $\mathcal{B}^{0}$ and the current $\mathcal{B}$ configurations rotating about the axis $\mathbf{i}_{1}$ and a close-up view of the cross section.

The kinematic description of the beam motion is provided in the Cartesian frame $\left\{C, \boldsymbol{e}_{1}, \boldsymbol{e}_{2}(t), \boldsymbol{e}_{3}(t)\right\}$ fixed to the beam reference, stress-free, configuration $\mathcal{B}^{0}$. This frame has its origin $C$ in the crosssection attached to the rotor (i.e., the beam root cross-section) center of mass and it is rotating rigidly with angular speed $\omega_{R}(t)$ about the vertical axis $\mathbf{i}_{1}$ of the fixed frame $\left\{O, \mathbf{i}_{1}, \mathbf{i}_{2}, \mathbf{i}_{3}\right\}$ (see Fig. 1). In particular, the $\boldsymbol{e}_{3}$-axis is collinear with the beam span, while $\boldsymbol{e}_{1}$ and $\boldsymbol{e}_{2}$ axes lay in the root cross-section plane, being, in turn, $\boldsymbol{e}_{1}$ collinear with the vertical fixed direction $\mathbf{i}_{1}$. Finally, the beam current configuration $\mathcal{B}$ is described through the orientation of the local inertial frame 
$\left\{C^{E}, \boldsymbol{b}_{1}(t), \boldsymbol{b}_{2}(t), \boldsymbol{b}_{3}(t)\right\}$ where, for symmetric beams, the mass center $C$ and the elastic center $C^{E}$ are coincident. Further details of the kinematic and mechanical modeling adopted in this work can be found in [15].

The rotating beam model, is parameterized in the space coordinate $s$, collinear with the beam reference center line and having its origin in the center of mass $C$ of the root cross-section and its end at the beam tip, while the motion of the beam is described at time $t$. The reference position vector of the space coordinate read as $\boldsymbol{r}^{\mathrm{o}}=\left(d_{3}+s\right) \boldsymbol{e}_{3}$, where $d_{3}$ is the rotor radius. On the other hand, the position vector in the current, dynamic configuration is $\boldsymbol{r}=\boldsymbol{r}^{\circ}+\boldsymbol{u}$, where $\boldsymbol{u}=u_{1} \boldsymbol{e}_{1}+u_{2} \boldsymbol{e}_{2}+u_{3} \boldsymbol{e}_{3}$ is the incremental, dynamic displacement of the beam centerline and $u_{1}, u_{2}$ and $u_{3}$ are the flapping, lagging, and axial displacements, respectively.

The orthogonal tensor $\boldsymbol{R}(s, t)$, whose expression is provided in [15], is then introduced to describe the orientation in space and time of the local inertial frame as $\boldsymbol{b}_{k}(s, t)=\boldsymbol{R}(s, t) \cdot \boldsymbol{e}_{k}(t)$. The latter, differentiated with respect to the space coordinate $s$, delivers the expression of the total curvature vector $\breve{\boldsymbol{\mu}}$ (i.e., $\partial_{s} \boldsymbol{b}_{k}(s, t)=\breve{\boldsymbol{\mu}}(s, t) \times \boldsymbol{b}_{k}(s, t)$, where $\partial$ stands for partial differentiation), while $\partial_{t} \boldsymbol{b}_{k}(s, t)=\breve{\boldsymbol{\omega}}(s, t) \times \boldsymbol{b}_{k}(s, t)$ gives the expression of the total angular velocity vector $\breve{\boldsymbol{\omega}}(s, t)$. The component forms of the curvature and the angular velocity vectors are reported in [15].

By introducing the vector $\boldsymbol{n}^{\circ}(s, t)$ of the prestressed contact force and the vectors $\boldsymbol{f}(s, t)$ and $c(s, t)$ of the incremental external force and couple per unit reference, respectively, the balance of linear and angular momentum provides the expressions of the beam equations of motion. The latter, describe the change of configuration between the prestressed equilibrium and the current, dynamic configuration in terms of incremental unknowns. Finally, the vectorial form of the equations of motion reads:

$$
\begin{aligned}
& \partial_{s} \boldsymbol{n}(s, t)+\boldsymbol{f}=\partial_{t} \breve{\boldsymbol{l}}-\partial_{t} \breve{\boldsymbol{l}}^{\mathrm{o}}, \\
& \partial_{s} \boldsymbol{m}(s, t)+\breve{\boldsymbol{\nu}}(s, t) \times \boldsymbol{n}(s, t)+\partial_{s} \boldsymbol{u}(s, t) \times \boldsymbol{n}^{\circ}(s, t)+\boldsymbol{c}=\partial_{t} \breve{\boldsymbol{h}}-\partial_{t} \breve{\boldsymbol{h}}^{\mathrm{o}},
\end{aligned}
$$

In Eq. (1), $\boldsymbol{n}=Q_{1} \boldsymbol{b}_{1}+Q_{2} \boldsymbol{b}_{2}+N \boldsymbol{b}_{3}$ and $\boldsymbol{m}=M_{1} \boldsymbol{b}_{1}+M_{2} \boldsymbol{b}_{2}+T \boldsymbol{b}_{3}$ represent the incremental contact forces and contact couples, respectively, where $\left(Q_{1}, Q_{2}\right)$ are the shear forces, $N$ is the axial force, $\left(M_{1}, M_{2}\right)$ are the bending moments, and $T$ is the torque. Moreover, $\breve{\boldsymbol{\nu}}(s, t)$ is the total stretch vector defined as $\breve{\boldsymbol{\nu}}(s, t) \equiv \partial_{s} \boldsymbol{r}=\breve{\eta}_{1}(s, t) \boldsymbol{b}_{1}(s, t)+\breve{\eta}_{2}(s, t) \boldsymbol{b}_{2}(s, t)+\breve{\nu}(s, t) \boldsymbol{b}_{3}(s, t)$, with $\breve{\eta}_{1}, \breve{\eta}_{2}$ being the total shear strains in the $\boldsymbol{b}_{1}(s, t)$ and $\boldsymbol{b}_{2}(s, t)$ directions, respectively, while $\breve{\nu}$ is the total stretch. Finally, $\partial_{t} \breve{\boldsymbol{l}}^{\mathrm{o}}, \partial_{t} \breve{\boldsymbol{l}}, \partial_{t} \breve{\boldsymbol{h}}^{\mathrm{o}}$ and $\partial_{t} \breve{\boldsymbol{h}}$ are the time rates of change of linear and angular momentum, respectively, in the prestressed (i.e., the parameters indicated by the superscript ${ }^{\circ}$ ) and the current configuration.

To study the interaction between the lagging and the axial motions, the beam dynamics are restrained into the $\left\{\boldsymbol{b}_{2}(s, t), \boldsymbol{b}_{3}(s, t)\right\}$ plane. In this case, the rotation tensor reads as follow:

$$
\mathbf{R}=\left[\begin{array}{ccc}
1 & 0 & 0 \\
0 & \cos \left(\theta_{1}\right) & \sin \left(\theta_{1}\right) \\
0 & -\sin \left(\theta_{1}\right) & \cos \left(\theta_{1}\right)
\end{array}\right]
$$


By neglecting the flapping displacement $u_{1}(s, t)$, the bending moment along $\boldsymbol{b}_{2}(s, t)$ and the torsional motion, the component form of the equations of the lagging and axial motion in symmetric rotating beams can be written as

$$
\begin{aligned}
& \partial_{s} Q_{2}(s, t) \cos \left(\theta_{1}(s, t)\right)-\partial_{s} N(s, t) \sin \left(\theta_{1}(s, t)\right)-\breve{\mu}_{1} Q_{2}(s, t) \sin \left(\theta_{1}(s, t)\right)-\breve{\mu}_{1} N(s, t) \cos \left(\theta_{1}(s, t)\right) \\
& =\rho A \partial_{t t} u_{2}(s, t)-2 \rho A \omega_{R} \partial_{t} u_{3}(s, t)-\rho A \omega_{R}^{2} u_{2}(s, t)-\rho A \dot{\omega}_{R} u_{3}(s, t), \\
& \partial_{s} Q_{2}(s, t) \sin \left(\theta_{1}(s, t)\right)+\partial_{s} N(s, t) \cos \left(\theta_{1}(s, t)\right)+\breve{\mu}_{1} Q_{2}(s, t) \cos \left(\theta_{1}(s, t)\right)-\breve{\mu}_{1} N(s, t) \sin \left(\theta_{1}(s, t)\right) \\
& =\rho A \partial_{t t} u_{3}(s, t)+2 \rho A \omega_{R} \partial_{t} u_{2}(s, t)-\rho A \omega_{R}^{2} u_{3}(s, t)+\rho A \dot{\omega}_{R} u_{2}(s, t), \\
& \partial_{s} M_{1}(s, t)-\breve{\nu} Q_{2}(s, t)+\partial_{s} u_{2}(s, t) N^{0}(s)=\rho J_{11}^{S} \partial_{t} \breve{\omega}_{1}-\rho J_{11}^{S} \dot{\omega}_{R},
\end{aligned}
$$

where $\rho A$ is the beam mass per unit reference length and $\rho J_{11}^{\mathrm{S}}$ is the mass moment of inertia of the cross section about the elastic center with respect to $\boldsymbol{b}_{1}(s, t)$. Finally, the boundary conditions at the root and at the tip of the beam read

$$
\begin{aligned}
& u_{2}(0, t)=0, u_{3}(0, t)=0, \quad \text { and } \quad \theta_{1}(0, t)=0, \\
& Q_{2}(L, t)=0, N(L, t)=0, \quad \text { and } \quad M_{1}(L, t)=0 .
\end{aligned}
$$

The same procedure proposed in [15] is here adopted to provide the curvature and the angular velocity vectors, respectively, as

$$
\breve{\mu}_{1}=\partial_{s} \theta_{1}, \breve{\mu}_{2}=0, \breve{\mu}_{3}=0,
$$

and

$$
\breve{\omega}_{1}=\partial_{t} \theta_{1}+\omega_{R}(t), \breve{\omega}_{2}=0, \breve{\omega}_{3}=0,
$$

respectively.

For the case of symmetric beams, the equations describing the prestress state and the corresponding boundary conditions reduce to $N^{0 \prime}(s)+\rho A \omega_{R}^{2}\left(d_{3}+s+u_{3}^{0}(s)\right)=0$ and $u_{3}^{0}(0)=0, \quad N^{0}(L)=0$ (see [15]), respectively, where $N^{0}(s)$ represents the prestress axial force, the ' denotes differentiation with respect to $s$.

By assuming an isotropic, linear elastic, constitutive behavior of the beam, the axial force and the bending moment can be then expressed in terms of the corresponding deformation modes as $N=E A(\nu-1)$ and $M_{1}=E J_{11}^{\mathrm{s}} \mu_{1}$, respectively, where $\nu$ and $\mu_{1}$ are the incremental stretch and curvature, respectively, while $E A$ is the axial stiffness and $E J_{11}^{S}$ is the lagging flexural stiffness. The slenderness of typical beams employed in helicopters or wind turbines is such to allow the assumption of unshearable beam. Therefore, the internal kinematic constraint $\breve{\eta}_{2}=0$ is introduced to neglect the effect of the shear strain along $\boldsymbol{b}_{2}$ and, hence, to obtain the relation between the bending rotation $\theta_{1}(s, t)$ and the components of the gradient of $\boldsymbol{u}(s, t)$ as $\theta_{1}=-\arctan \left[\partial_{s} u_{2} /\left(\partial_{s} u_{3}+1 / \nu_{\text {inv }}^{0}(s)\right)\right]$, where $\nu_{i n v}^{0}(s)=1 /\left(1+u_{3}^{\prime 0}(s)\right)$ is the inverse of the prestress stretch. Furthermore, the total stretch reads $\breve{\nu}=\left[\left(\partial_{s} u_{3}+1 / \nu_{i n v}^{0}(s)\right)^{2}+\partial_{s} u_{2}^{2}\right]^{1 / 2}$, while the expression of the shear force $Q_{2}(s, t)$ can be 
obtained from Eqs. $(3)_{3}$ and substituted in Eqs. $(3)_{1-2}$ to finally deliver the equations governing the lagging and the axial motions.

\section{Nondimensionalization}

By introducing the following nondimensional parameters: $\hat{s}=s / L, \hat{t}=\omega_{0} t, \hat{u}_{2}=u_{2} / L, \hat{u}_{3}=$ $u_{3} / L$, where $\omega_{0}=\sqrt{\frac{E J_{22}^{S}}{\rho A L^{4}}}$, the nondimensional form of the lagging and axial equations of motion, together with the associated boundary conditions at $s=1$, read

$$
\begin{gathered}
\mathbf{I} \cdot \ddot{\mathbf{u}}+\mathbf{G} \cdot \dot{\mathbf{u}}+\mathbf{L} \cdot \mathbf{u}+\mathbf{i}_{1}^{(2)}(\dot{\mathbf{u}}, \dot{\mathbf{u}})+\mathbf{i}_{2}^{(2)}(\mathbf{u}, \ddot{\mathbf{u}})+ \\
+\mathbf{i}_{1}^{(3)}(\mathbf{u}, \dot{\mathbf{u}}, \dot{\mathbf{u}})+\mathbf{i}_{2}^{(3)}(\mathbf{u}, \mathbf{u}, \ddot{\mathbf{u}}) \\
+\mathbf{n}^{(2)}(\mathbf{u}, \mathbf{u})+\mathbf{n}^{(3)}(\mathbf{u}, \mathbf{u}, \mathbf{u})=0, \\
\left\{I_{B C 21}\left(\ddot{u}_{2}\right)+L_{B C 21}\left(u_{2}\right)+i_{B C 21,1}^{(2)}(\dot{u}, \dot{u})+i_{B C 21,2}^{(2)}(u, \ddot{u})+i_{B C 21,1}^{(3)}(u, \dot{u}, \dot{u})+i_{B C 21,2}^{(3)}(u, u, \ddot{u})\right. \\
\left.+n_{B C 21}^{(2)}(u, u)+n_{B C 21}^{(3)}(u, u, u)\right\}\left.\right|_{s=1}=0, \\
\left.\left\{L_{B C 22}\left(u_{2}\right)+n_{B C 22}^{(2)}(u, u)+n_{B C 22}^{(3)}(u, u, u)\right\}\right|_{s=1}=0, \\
\left.\left\{L_{B C 22}\left(u_{3}\right)+n_{B C 3}^{(2)}(u, u)+n_{B C 3}^{(3)}(u, u, u)\right\}\right|_{s=1}=0,
\end{gathered}
$$

where $\mathbf{u}$ represents the 2 by 1 operator including the axial and the lagging components of the beam motion, i.e., $\mathbf{u}=\left[u_{2}(s, t) u_{3}(s, t)\right]^{\top}$, while $\mathbf{I}, \mathbf{G}$ and $\mathbf{L}$ are the linear inertia and the gyroscopic and elastic stiffness operators, respectively, whose expressions are given in Appendix I. Moreover, $\mathbf{i}_{0}^{(2)}$, $\mathbf{i}_{1}^{(2)}, \mathbf{i}_{2}^{(2)}$ and $\mathbf{i}_{0}^{(3)}, \mathbf{i}_{1}^{(3)}, \mathbf{i}_{2}^{(3)}$ are the quadratic and the cubic inertial terms, respectively, and $\mathbf{n}^{(2)}$, and $\mathbf{n}^{(3)}$ represent the quadratic and the cubic stiffness, respectively. The quadratic operators are defined in Appendix II, while, for the sake of brevity, the third order operators are omitted.

Further nondimensional parameters adopted are: $\lambda=\frac{\omega_{R}}{\omega_{0}}, \alpha_{12}=\frac{E A L^{2}}{E J_{11}^{S}}, \alpha_{22}=\frac{E A L^{2}}{E J_{22}^{S}}, \alpha_{32}=\frac{E A L^{2}}{E J_{33}^{S}}$ and $G J_{33}^{S}=\frac{E J_{33}^{S}}{2(1+\bar{\nu})}$, where $\bar{\nu}$ is the Poisson coefficient. Accordingly, for the case of symmetric beams the only relations which provide the prestressed configuration and the associated boundary conditions, respectively, are simplified to $\alpha_{22} u_{3}^{0 \prime \prime}(s)+\lambda^{2}\left(r+s+u_{3}^{0}(s)\right)=0$ and $u_{3}^{0}(0)=$ $0, \quad N^{0}(1)=0$. Thus, the prestressed axial configuration has the following expression: $u_{3}^{0}(s)=$ $\sin \left(\lambda_{a} s\right) \frac{\left(\sin \left(\lambda_{a}\right)\right) \lambda r+\sqrt{\alpha_{22}}}{\left(\cos \left(\lambda_{a}\right)\right) \lambda}+\cos \left(\lambda_{a} s\right) r-s-r$, where $\lambda_{a}=\frac{\lambda}{\sqrt{\alpha_{22}}}$ and $r=\frac{d_{3}}{L}$.

\section{Nonlinear free vibrations of the rotating beam}

The direct method of multiple scales is adopted to perform a perturbation analysis of the equations of motion Eqs. (7) and (8). The asymptotic expansion of the lagging and the axial displacements is considered in the form [26]: $u_{2}\left(s, T_{0}, T_{1}, T_{2}\right)=\varepsilon u_{2,0}\left(s, T_{0}, T_{1}, T_{2}\right)+\varepsilon^{2} u_{2,1}\left(s, T_{0}, T_{1}, T_{2}\right)+$ $\varepsilon^{3} u_{2,2}\left(s, T_{0}, T_{1}, T_{2}\right)$ and $u_{3}\left(s, T_{0}, T_{1}, T_{2}\right)=\varepsilon u_{3,0}\left(s, T_{0}, T_{1}, T_{2}\right)+\varepsilon^{2} u_{3,1}\left(s, T_{0}, T_{1}, T_{2}\right)+\varepsilon^{3} u_{3,2}\left(s, T_{0}, T_{1}, T_{2}\right)$, where $\varepsilon$ is a non-physical parameter used for ordering the nonlinearity of the system, and $T_{0}$ is the nondimensional fast time scale, while and $T_{1}$ and $T_{2}$ are the nondimensional slow time scales [24]. By substituting the third-order expansion of the solution into Eqs. (7) and (8), a hierarchy of problems 
of order $\varepsilon, \varepsilon^{2}$, and $\varepsilon^{3}$, respectively, is obtained by equating to zero coefficients of like powers of $\varepsilon$. Therefore, the lowest three perturbation problems are given by

Order $\varepsilon$

$$
\begin{gathered}
-I_{22} D_{0}^{2}\left[u_{2,0}\right]+G_{23} D_{0}\left[u_{3,0}\right]+L_{22}\left[u_{2,0}\right]=0, \\
-I_{33} D_{0}^{2}\left[u_{3,0}\right]+G_{32} D_{0}\left[u_{2,0}\right]+L_{33}\left[u_{3,0}\right]=0, \\
-I_{B C 21} D_{0}^{2}\left[u_{2,0}\right]+L_{B C 21}\left[u_{2,0}\right]=0, \\
L_{B C 22}\left[u_{2,0}\right]=0 \\
L_{B C 3}\left[u_{3,0}\right]=0
\end{gathered}
$$

Order $\varepsilon^{2}$

$$
\begin{gathered}
-I_{22} D_{0}^{2}\left[u_{2,1}\right]+G_{23} D_{0}\left[u_{3,1}\right]+L_{22}\left[u_{2,1}\right]=\operatorname{RHS}\left(O_{E q 2}^{(2)}\right), \\
-I_{33} D_{0}^{2}\left[u_{3,1}\right]+G_{32} D_{0}\left[u_{2,1}\right]+L_{33}\left[u_{3,1}\right]=\operatorname{RHS}\left(O_{E q 3}^{(2)}\right), \\
-I_{B C 21} D_{0}^{2}\left[u_{2,1}\right]+L_{B C 21}\left[u_{2,1}\right]=\operatorname{RHS}\left(O_{B C 21}^{(2)}\right), \\
L_{B C 22}\left[u_{2,1}\right]=\operatorname{RHS}\left(O_{B C 22}^{(2)}\right), \\
L_{B C 3}\left[u_{3,1}\right]=\operatorname{RHS}\left(O_{B C 3}^{(2)}\right),
\end{gathered}
$$

Order $\varepsilon^{3}$

$$
\begin{gathered}
-I_{22} D_{0}^{2}\left[u_{2,2}\right]+G_{23} D_{0}\left[u_{3,2}\right]+L_{22}\left[u_{2,2}\right]=\operatorname{RHS}\left(O_{E q 2}^{(3)}\right), \\
-I_{33} D_{0}^{2}\left[u_{3,2}\right]+G_{32} D_{0}\left[u_{2,2}\right]+L_{33}\left[u_{3,2}\right]=\operatorname{RHS}\left(O_{E q 3}^{(3)}\right), \\
-I_{B C 21} D_{0}^{2}\left[u_{2,2}\right]+L_{B C 21}\left[u_{2,2}\right]=\operatorname{RHS}\left(O_{B C 21}^{(3)}\right), \\
L_{B C 22}\left[u_{2,2}\right]=\operatorname{RHS}\left(O_{B C 22}^{(3)}\right), \\
L_{B C 3}\left[u_{3,2}\right]=\operatorname{RHS}\left(O_{B C 3}^{(3)}\right),
\end{gathered}
$$

where the expressions of the right-hand side terms are omitted for the sake of brevity and simply named RHS.

The first order problem, i.e. Eqs. (9) and (10), provides the linear free vibrations of the beam and its solution can be written in the form

$$
\begin{aligned}
& u_{2,0}=\psi_{2, k}(s)\left[A_{k}\left(T_{1}, T_{2}\right) e^{i \omega_{2, k} T_{0}}+c c\right], \\
& u_{3,0}=\psi_{3, k}(s)\left[A_{k}\left(T_{1}, T_{2}\right), e^{i \omega_{2, k} T_{0}}+c c\right]
\end{aligned}
$$

where $\psi_{2, k}$ and $\psi_{3, k}$ are the $k$ th lagging and axial mode shapes, respectively, which are determined by employing the Galerkin discretization approach [25], while $A_{k}\left(T_{1}, T_{2}\right)$ and $\omega_{2, k}$ are the $k$ th complex amplitude and lagging-axial natural frequency, respectively. Finally, $i$ is the imaginary unit and $c c$ stands for complex conjugate.

The first order solution is then substituted into the right-hand side of the $\varepsilon^{2}$ problem, i.e. Eqs. (11) and (12), to calculate the second order solution. To eliminate terms causing the appearance 
of secular terms, the following solvability condition is enforced according to Fredholm's alternative Theorem:

$$
\begin{aligned}
& \int_{0}^{1}\left[\psi_{2, k}(s) \cdot C_{1,2}\left(s, T_{1}, T_{2}\right)+\psi_{3, k}(s) \cdot C_{1,3}\left(s, T_{1}, T_{2}\right)\right] d s \\
& \quad-C_{1, B C 12}\left(T_{1}, T_{2}\right) \cdot \psi_{2, k}(1)+C_{1, B C 22}\left(T_{1}, T_{2}\right) \cdot \psi_{2, k}^{\prime}(1)-C_{1, B C 3}\left(T_{1}, T_{2}\right) \cdot \psi_{3, k}(1)=0
\end{aligned}
$$

where $C_{1,2}\left(s, T_{1}, T_{2}\right), C_{1,3}\left(s, T_{1}, T_{2}\right)$ are the coefficients of the terms proportional to $e^{i \omega_{2, k} T_{0}}$ in the RHS of Eq. (11), while $C_{1, B C 21}\left(T_{1}, T_{2}\right), C_{1, B C 22}\left(T_{1}, T_{2}\right)$ and $C_{1, B C 3}\left(T_{1}, T_{2}\right)$ refer to the RHS of Eq. (12). Therefore, the modulation in $T_{1}$ of the amplitude $A_{k}\left(T_{1}, T_{2}\right)$ is provided by the equation

$$
\left[\frac{2\left(i \omega_{2, k} \xi_{1} \alpha_{12}-2 i \omega_{2, k} \xi_{2}-\lambda \xi_{3} \alpha_{12}\right)}{\alpha_{12}}+2 i \omega_{2, k} \xi_{4}+2 \lambda \xi_{3}-i C_{1}\right] D_{1} A_{k}\left(T_{1}, T_{2}\right)=0
$$

where $C_{1}=-\left.2\left(\left.\psi_{2, k}^{\prime}\right|_{s=1}\right) \omega_{2, k} \psi_{2, k}\right|_{s=1} / \alpha_{12}$ and the $\xi_{i}$ coefficients are reported in Appendix III. As clear in the modulation equation, Eq. (17), the complex amplitude $A_{k}$ does not depend on $T_{1}$, that is, $A_{k}\left(T_{1}, T_{2}\right)=A_{k}\left(T_{2}\right)$. Therefore, the particular solution of the second order problem can be written as

$$
\begin{aligned}
& u_{2,1}\left(s, T_{0}, T_{1}, T_{2}\right)=h_{21}(s) A_{k}\left(T_{2}\right) \bar{A}_{k}\left(T_{2}\right)+h_{22, R}(s) e^{2 i \omega_{2, k} T_{0}} A_{k}\left(T_{2}\right)^{2}+i h_{22, i}(s) e^{2 i \omega_{2, k} T_{0}} A_{k}\left(T_{2}\right)^{2}+c c \\
& u_{3,1}\left(s, T_{0}, T_{1}, T_{2}\right)=h_{31}(s) A_{k}\left(T_{2}\right) \bar{A}_{k}\left(T_{2}\right)+h_{32, R}(s) e^{2 i \omega_{2, k} T_{0}} A_{k}\left(T_{2}\right)^{2}+i h_{32, i}(s) e^{2 i \omega_{2, k} T_{0}} A_{k}\left(T_{2}\right)^{2}+c c
\end{aligned}
$$

where $h_{22, R}(s), h_{32, i}(s), h_{32, R}(s), h_{22, i}(s), h_{21}(s)$ and $h_{31}(s)$ are functions of the space coordinate $s$ and are calculated by solving, by means of the Galerkin approach, the following ordinary differential equations

$$
\begin{gathered}
\lambda^{2} h_{22, R}-3 \alpha_{22}\left(\nu_{i n v}^{0}{ }^{\prime \prime}\right) \nu_{i n v}^{0} h_{22, R}^{\prime \prime} / \alpha_{12}-\alpha_{22}\left(\nu_{i n v}^{0}\right)\left(\nu_{i n v}^{0}{ }^{\prime \prime}\right) h_{22, R}^{\prime} / \alpha_{12}-2 \alpha_{22}\left(\nu_{i n v}^{0}\right)^{2} h_{22, R}^{\prime \prime} / \alpha_{12} \\
-\alpha_{22} \nu_{i n v}^{0}{ }^{2} h_{22, R}^{\prime \prime \prime \prime} / \alpha_{12}+4 h_{22, R} \omega_{2, k}^{2}+\alpha_{22}\left(N_{0}^{\prime}\right) \nu_{i n v}^{0} h_{22, R}^{\prime}+\alpha_{22} N_{0} \nu_{i n v}^{0} h_{22, R}^{\prime \prime} \\
+\alpha_{22} N_{0}\left(\nu_{i n v}^{0}{ }^{\prime}\right) h_{22, R}^{\prime}-8\left(\nu_{i n v}^{0}{ }^{\prime}\right) \nu_{i n v}^{0} h_{22, R}^{\prime} \omega_{2, k}^{2} / \alpha_{12}-4 \alpha_{22}\left(\nu_{i n v}^{0}{ }^{\prime}\right) \nu_{i n v}^{0} h_{22, R}^{\prime \prime \prime} / \alpha_{12} \\
-\alpha_{22}\left(\nu_{i n v}^{0}{ }^{\prime \prime \prime}\right) \nu_{i n v}^{0} h_{22, R}^{\prime} / \alpha_{12}-4 \nu_{i n v}^{0}{ }^{2} h_{22, R}^{\prime \prime} \omega_{2, k}^{2} / \alpha_{12}-4 \lambda h_{32, i} \omega_{2, k}=f_{22 R 32 i}, \\
h_{32, i} \lambda^{2}-4 h_{22, R} \lambda \omega_{2, k}+4 h_{32, i} \omega_{2, k}^{2}+h_{32, i}^{\prime \prime} \alpha_{22}=0 \\
\lambda^{2} h_{22, i}-4 \alpha_{22}\left(\nu_{i n v}^{0}{ }^{\prime}\right) \nu_{i n v}^{0} h_{22, i}^{\prime \prime \prime} / \alpha_{12}-\alpha_{22}\left(\nu_{i n v}^{0}{ }^{\prime}\right)\left(\nu_{i n v}^{0}{ }^{\prime \prime}\right) h_{22, i}^{\prime} / \alpha_{12}-2 \alpha_{22}\left(\nu_{i n v}^{0}{ }^{\prime}\right)^{2} h_{22, i}^{\prime \prime} / \alpha_{12} \\
-\alpha_{22} \nu_{i n v}^{0}{ }^{2} h_{22, i}^{\prime \prime \prime} / \alpha_{12}-4 \nu_{i n v}^{0}{ }^{2} h_{22, i}^{\prime \prime} \omega_{2, k}^{2} / \alpha_{12}-\alpha_{22}\left(\nu_{i n v}^{0}{ }^{\prime \prime \prime}\right) \nu_{i n v}^{0} h_{22, i}^{\prime} / \alpha_{12} \\
+4 h_{22, i} \omega_{2, k}^{2}-3 \alpha_{22}\left(\nu_{i n v}^{0}{ }^{\prime \prime}\right) \nu_{i n v}^{0} h_{22, i}^{\prime \prime} / \alpha_{12}-8\left(\nu_{i n v}^{0}{ }^{\prime}\right) \nu_{i n v}^{0} h_{22, i}^{\prime} \omega_{2, k}^{2} / \alpha_{12} \\
+\alpha_{22} N_{0} \nu_{i n v}^{0} h_{22, i}^{\prime \prime}+\alpha_{22} N_{0}\left(\nu_{i n v}^{0}{ }^{\prime}\right) h_{22, i}^{\prime}+\alpha_{22}\left(N_{0}^{\prime}\right) \nu_{i n v}^{0} h_{22, i}^{\prime}+4 \lambda h_{32, R} \omega_{2, k}=0, \\
\lambda^{2} h_{32, R}+4 h_{22, i} \lambda \omega_{2, k}+4 h_{32, R} \omega_{2, k}^{2}+h_{32, R}^{\prime \prime} \alpha_{22}=f_{32 R 22 i}(s),
\end{gathered}
$$




$$
\begin{gathered}
\lambda^{2} h_{21}-\alpha_{22} \nu_{i n v}^{0}{ }^{2} h_{21}^{\prime \prime \prime \prime} / \alpha_{12}-2 \alpha_{22}\left(\nu_{i n v}^{0}{ }^{\prime}\right)^{2} h_{21}^{\prime \prime} / \alpha_{12}-\alpha_{22}\left(\nu_{i n v}^{0}{ }^{\prime \prime \prime}\right) \nu_{i n v}^{0} h_{21}^{\prime} / \alpha_{12} \\
-4 \alpha_{22}\left(\nu_{i n v}^{0}{ }^{\prime}\right) \nu_{i n v}^{0} h_{21}^{\prime \prime \prime} / \alpha_{12}-3 \alpha_{22}\left(\nu_{i n v}^{0}{ }^{\prime \prime}\right) \nu_{i n v}^{0} h_{21}^{\prime \prime} / \alpha_{12}-\alpha_{22}\left(\nu_{i n v}^{0}{ }^{\prime}\right)\left(\nu_{i n v}^{0}{ }^{\prime \prime}\right) h_{21}^{\prime} / \alpha_{12} \\
+\alpha_{22}\left(N_{0}^{\prime}\right) \nu_{i n v}^{0} h_{21}^{\prime}+\alpha_{22} N_{0} \nu_{i n v}^{0} h_{21}^{\prime \prime}+\alpha_{22} N_{0}\left(\nu_{i n v}^{0}{ }^{\prime}\right) h_{21}^{\prime}=f_{21}(s), \\
\lambda^{2} h_{31}+\alpha_{22} h_{31}^{\prime \prime}=f_{31}(s),
\end{gathered}
$$

where all the right-hand side is omitted for the sake of brevity.

By now substituting the expressions of the first- and second-order solutions into the third-order problem (i.e., Eqs. (13) and (14)), eliminating terms causing the appearance of secular terms delivers the following modulation equation in time $T_{2}$

$$
2 i \omega_{2, k} \Gamma_{1, k} D_{2} A_{k}\left(T_{2}\right)+\left(i \Gamma_{2, k, i}+\Gamma_{2, k, R}\right) \bar{A}_{k}\left(T_{2}\right) A_{k}\left(T_{2}\right)^{2}=0,
$$

where the coefficients $\Gamma$ are defined in Appendix IV. The time rate-of-change of the complex amplitude $A_{k}\left(T_{2}\right)$ can be then written as

$$
D_{2} A_{k}\left(T_{2}\right)=\left(i \gamma_{k, i}+\gamma_{k, R}\right) \bar{A}_{k}\left(T_{2}\right) A_{k}\left(T_{2}\right)^{2}
$$

where the expressions of the coefficients $\gamma$ are reported in Appendix IV.

By introducing the polar form of the $k$ th amplitude as $A_{k}\left(T_{2}\right)=\frac{1}{2} a_{k}\left(T_{2}\right) e^{i \beta_{k}\left(T_{2}\right)}$, where $a_{k}\left(T_{2}\right)$ and $\beta_{k}\left(T_{2}\right)$ are the real amplitude and the phase, respectively, and substituting it into the modulation equation, separating real and imaginary parts yield

$$
\begin{aligned}
& D_{2} a_{k}\left(T_{2}\right)=0, \\
& a_{k}\left(T_{2}\right) D_{2} \beta_{k}\left(T_{2}\right)=\frac{1}{4} a_{k}\left(T_{2}\right)^{3} \gamma_{k, i},
\end{aligned}
$$

where $\gamma_{k, i} \equiv \gamma_{k}$ is the so-called the effective nonlinearity coefficient which characterize the type of nonlinearity of each mode. Therefore, the solution of Eqs. (27) reads $a_{k}\left(T_{2}\right)=a_{k}^{0}$ and $\beta_{k}\left(T_{2}\right)=$ $\frac{1}{4} a_{k}^{02} \gamma_{k} T_{2}+\beta_{k}^{0}$, where $a_{k}^{0}$ and $\beta_{k}^{0}$ are defined through the initial conditions.

The solution, up to the second-order approximation, can be the written as

$$
\begin{gathered}
u_{2}=\psi_{2, k}(s) a_{k}^{0} \cos \left(\omega_{2, k}^{N L} t+\beta_{k}^{0}\right)+\frac{1}{2} h_{21}(s) a_{k}^{02}+\frac{1}{2} h_{22, R}(s) a_{k}^{02} \cos \left(2 \omega_{2, k}^{N L} t+2 \beta_{k}^{0}\right) \\
-\frac{1}{2} h_{22, i}(s) a_{k}^{02} \sin \left(2 \omega_{2, k}^{N L} t+2 \beta_{k}^{0}\right), \\
u_{3}=\psi_{3, k}(s) a_{k}^{0} \cos \left(\omega_{2, k}^{N L} t+\beta_{k}^{0}\right)+\frac{1}{2} h_{31}(s) a_{k}^{02}+\frac{1}{2} h_{32, R}(s) a_{k}^{02} \cos \left(2 \omega_{2, k}^{N L} t+2 \beta_{k}^{0}\right) \\
-\frac{1}{2} h_{32, i}(s) a_{k}^{02} \sin \left(2 \omega_{2, k}^{N L} t+2 \beta_{k}^{0}\right),
\end{gathered}
$$

where $\omega_{2, k}^{N L}=\omega_{2, k}+\frac{1}{4} a_{k}^{02} \gamma_{k}$ is the $k$ th lagging-axial nonlinear frequency and the parameter $\gamma_{k}$ is the nonlinearity coefficient. 


\section{Principal parametric resonance}

Principal parametric resonance may occur when the beam angular velocity is modulated in time by a small, harmonic oscillation and the frequency of the oscillation is twice one of the natural frequency of the beam [26]. Therefore, to study the above mentioned phenomenon, the rotational speed $\lambda$ is assumed to have the following expression: $\lambda(t)=\lambda(1+\delta \cos (\Omega t))$, where $\delta$ and $\Omega$ are the amplitude and frequency, respectively, of the parametric excitation. Moreover, the frequency of the oscillating part of $\lambda$ is expressed as $\Omega=2 \omega_{2, k}+\varepsilon^{2} \sigma$, where $\sigma$ is the detuning parameter. The linear damping forces are introduced through the classical Rayleigh formulation in the form $-2 \zeta_{2} \omega_{2, k} \partial_{t} u_{2}(s, t)$ and $-2 \zeta_{3} \omega_{2, k} \partial_{t} u_{3}(s, t)$. For the case of weak damping, the dissipative terms can be then rescaled so as to be proportional to $\varepsilon^{2}$, furthermore, also the effects due to the excitation provided by the periodic part $\delta$ of the angular speed can be assumed to be of higher order and, then, rescaled as $\delta=\varepsilon^{2} \delta$.

The same asymptotic procedure discussed in section 3 is adopted to study the principal parametric resonance. Although, since the dissipative terms and the modulation of the angular speed are assumed as proportional to $\varepsilon^{2}$, their contribution appears only at the order $\varepsilon^{3}$ of the asymptotic expansion, therefore, it turns out that the solutions of the first and the second perturbation corresponds to those found in section 3, i.e., Eq. (15) and Eq. (18). By substituting the latter into the third-order problem of the parametric excitation case, eliminating terms causing the appearance of secular terms delivers the following modulation equation in time $T_{2}$

$2 i \omega_{2, k} \Gamma_{1, k} A_{k}^{\prime}\left(T_{2}\right)+\left(i \Gamma_{2, k, i}+\Gamma_{2, k, R}\right) \bar{A}_{k}\left(T_{2}\right) A_{k}\left(T_{2}\right)^{2}+2 \lambda^{2} \delta \Gamma_{3, k} \bar{A}_{k}\left(T_{2}\right) e^{i \sigma T_{2}}+2 i \zeta_{2, k} \omega_{2, k}^{2} A_{k}\left(T_{2}\right)=0$,

where the prime, here and henceforth, will be adopted to indicate differentiation with respect to time $T_{2}$ and the expressions of parameters $\Gamma$ are given in Appendix IV. The time rate-of-change of the complex amplitude $A_{k}\left(T_{2}\right)$ can be then written as

$$
A_{k}^{\prime}\left(T_{2}\right)=\left(i \gamma_{k, i}+\gamma_{k, R}\right) \bar{A}_{k}\left(T_{2}\right) A_{k}\left(T_{2}\right)^{2}+i \gamma_{1, k} \bar{A}_{k}\left(T_{2}\right) e^{i \sigma T_{2}}+\gamma_{2, k} A_{k}\left(T_{2}\right)
$$

where coefficients $\gamma$ are reported in Appendix IV.

By substituting the polar form of the $k$ th amplitude $A_{k}\left(T_{2}\right)=(1 / 2) a_{k}\left(T_{2}\right) e^{i \beta_{k}\left(T_{2}\right)}$, into the modulation equation Eq. (30), separating real and imaginary parts yield and introducing the relative phase $\theta_{k}\left(T_{2}\right)=\sigma T_{2}-2 \beta_{k}\left(T_{2}\right)$, the equations governing the time evolution of the real amplitude and the relative phase can be written as

$$
\begin{aligned}
& a_{k}^{\prime}\left(T_{2}\right)=\frac{1}{4} a_{k}\left(T_{2}\right)^{3} \gamma_{k, R}-a_{k}\left(T_{2}\right) \sin \left(\theta_{k}\left(T_{2}\right)\right) \gamma_{1, k}+\gamma_{2, k} a_{k}\left(T_{2}\right), \\
& a_{k}\left(T_{2}\right) \theta_{k}^{\prime}\left(T_{2}\right)=-\frac{1}{2} a_{k}\left(T_{2}\right)^{3} \gamma_{k, i}-2 a_{k}\left(T_{2}\right) \gamma_{1, k} \cos \left(\theta_{k}\left(T_{2}\right)\right)+a_{k}\left(T_{2}\right) .
\end{aligned}
$$




\subsection{Stability analysis}

Modulation equations reported in Eq. (31) have both trivial and nontrivial solutions which must be calculated separately. The stability of the nontrivial steady state solution of the amplitude and phase modulation equations is studied through the eigenvalues of the Jacobian of Eq. (31) which leads to the following characteristic equation:

$$
\lambda^{2}+\left(-a_{k}^{2} \gamma_{k, R}-2 \gamma_{2, k}\right) \lambda-\frac{1}{2} a_{k}^{2} \gamma_{k, i} \sigma+\frac{1}{4} a_{k}^{4} \gamma_{k, R}^{2}+a_{k}^{2} \gamma_{2, k} \gamma_{k, R}+\frac{1}{4} a_{k}^{4} \gamma_{k, i}^{2}=0
$$

Following the Routh-Hurwitz stability criterion, the subsequent relations must be satisfied to ensure the stability of the steady state solution:

$$
\begin{aligned}
& -a_{k}^{2} \gamma_{k, R}-2 \gamma_{2, k}>0 \\
& -\frac{1}{2} a_{k}^{2} \gamma_{k, i} \sigma+\frac{1}{4} a_{k}^{4} \gamma_{k, R}^{2}+a_{k}^{2} \gamma_{2, k} \gamma_{k, R}+\frac{1}{4} a_{k}^{4} \gamma_{k, i}^{2}>0
\end{aligned}
$$

To study the stability of the trivial solution, the Cartesian form of the amplitude, i.e. $A_{k}\left(T_{2}\right)=$ $\left(p_{k}\left(T_{2}\right)+i q_{k}\left(T_{2}\right)\right) e^{\frac{1}{2} i \sigma T_{2}}$, is substituted into Eq. (30), so as to obtain the following equations

$$
\begin{aligned}
& p_{k}^{\prime}\left(T_{2}\right)=\gamma_{k, R} p\left(T_{2}\right)^{3}-\gamma_{k, i} p\left(T_{2}\right)^{2} q\left(T_{2}\right)+\left(\gamma_{k, R} q\left(T_{2}\right)^{2}+\gamma_{2, k}\right) p\left(T_{2}\right)-\gamma_{k, i} q\left(T_{2}\right)^{3}+\left(\frac{1}{2} \sigma+\gamma_{1, k}\right) q\left(T_{2}\right), \\
& q_{k}^{\prime}\left(T_{2}\right)=\gamma_{k, i} p\left(T_{2}\right)^{3}+\gamma_{k, R} p\left(T_{2}\right)^{2} q\left(T_{2}\right)+\left(\gamma_{k, i} q\left(T_{2}\right)^{2}-\frac{1}{2} \sigma+\gamma_{1, k}\right) p\left(T_{2}\right)+\gamma_{k, R} q\left(T_{2}\right)^{3}+\gamma_{2, k} q\left(T_{2}\right),
\end{aligned}
$$

and the characteristic equation derived from the Jacobian reads

$$
\lambda^{2}-2 \lambda \gamma_{2, k}+\frac{1}{4} \sigma^{2}+\gamma_{2, k}^{2}-\gamma_{1, k}^{2}=0
$$

Finally, the Routh-Hurwitz stability criterion yields

$$
\begin{aligned}
& -2 \gamma_{2, k}>0, \\
& \frac{1}{4} \sigma^{2}+\gamma_{2, k}^{2}-\gamma_{1, k}^{2}>0 .
\end{aligned}
$$

By substituting the first- and second-order solutions provided by Eqs. (15) and (18) into the asymptotic expansion of the beam displacements and by letting $\varepsilon=1$, the second order form of the lagging and axial displacements can be written as

$$
\begin{array}{r}
u_{2}=\psi_{2, k}(s) a_{k}(t) \cos \left(\beta_{k}(t)+\omega_{2, k} t\right)+\frac{1}{2} h_{21}(s) \\
a_{k}(t)^{2}+\frac{1}{2} h_{22, R}(s) a_{k}(t)^{2} \cos \left(2 \omega_{2, k} t+2 \beta_{k}(t)\right) \\
-\frac{1}{2} h_{22, i}(s) a_{k}(t)^{2} \sin \left(2 \omega_{2, k} t+O\left(\varepsilon^{3}\right),\right. \\
u_{3}=\psi_{3, k}(s) a_{k}(t) \cos \left(\beta_{k}(t)+\omega_{2, k} t\right)+\frac{1}{2} h_{31}(s) \\
a_{k}(t)^{2}+\frac{1}{2} h_{32, R}(s) a_{k}(t)^{2} \cos \left(2 \omega_{2, k} t+2 \beta_{k}(t)\right) \\
-\frac{1}{2} h_{32, i}(s) a_{k}(t)^{2} \sin \left(2 \omega_{2, k} t+2 \beta_{k}(t)\right)+O\left(\varepsilon^{3}\right) .
\end{array}
$$




\section{Numerical simulations via Newmark- $\beta$ method}

In this section, the Newmark- $\beta$ method [27] is implemented so as to calculate the dynamic response of the rotating beam undergoing principal parametric resonance via direct time integration of the linearized form of the equations of motion discretized via Galerkin approach [25]. In particular, this discretization procedure is applied to the lagging-axial equations so as to obtain the following discrete form of the equations of motion

$$
\begin{aligned}
& M_{2,2} \ddot{q}_{2, k}(t)+C_{2,3} \dot{q}_{3, k}(t)+K_{2,2} q_{2, k}(t)+K_{2,3} q_{3, k}(t)=0, \\
& M_{3,3} \ddot{q}_{3, k}(t)+C_{3,2} \dot{q}_{2, k}(t)+K_{3,3} q_{3, k}(t)+K_{3,2} q_{2, k}(t)=0,
\end{aligned}
$$

where, $q_{2, k}(t)$ and $q_{3, k}(t)$ are the $k$ th lagging and axial generalized coordinates, respectively, while

$$
\begin{aligned}
& M_{2,2}=\left(\alpha_{12} \xi_{1}-\xi_{5}-2 \xi_{6}\right) / \alpha_{12} \quad C_{2,3}=C_{2,3, \mathrm{cons}}+C_{2,3, \cos } \cos (\Omega t) \\
& K_{2,2}=K_{2,2, \mathrm{cons}}+K_{2,2, \cos } \cos (\Omega t)+K_{2,2, \cos ^{2}} \cos (\Omega t)^{2} \quad K_{2,3}=\xi_{3} \lambda \delta \Omega \sin (\Omega t),
\end{aligned}
$$

and

$$
\begin{aligned}
& M_{3,3}=\xi_{4} \quad C_{3,2}=C_{3,2, \operatorname{cons}}+C_{3,2, \cos } \cos (\Omega t) \quad K_{3,2}=-\xi_{3} \lambda \delta \Omega \sin (\Omega t) \\
& K_{3,3}=K_{3,3, \mathrm{cons}}+K_{3,3, \cos } \cos (\Omega t)+K_{2,2, \cos ^{2}} \cos (\Omega t)^{2},
\end{aligned}
$$

where the expressions of coefficients $C_{i, j, \mathrm{cons}}, C_{i, j, \mathrm{cos}}, K_{i, j, \mathrm{cons}}, K_{i, j, \mathrm{cos}}, K_{i, j, \cos ^{2}}$ and $K_{i, j, \mathrm{sin}}$ are given in Appendix V. Hence, the lagging-axial equations can be reorganized in matrix form as:

$$
\mathbf{M} \ddot{\mathbf{q}}+\mathbf{C} \dot{\mathbf{q}}+\mathbf{K q}=0
$$

where the mass $\mathbf{M}$, the Coriolis $\mathbf{C}$ and the stiffness $\mathbf{K}$ matrices are

$$
\mathbf{M}=\left[\begin{array}{cc}
M_{2,2} & 0 \\
0 & M_{3,3}
\end{array}\right], \mathbf{C}=\left[\begin{array}{cc}
0 & C_{2,3} \\
C_{3,2} & 0
\end{array}\right], \mathbf{K}=\left[\begin{array}{ll}
K_{2,2} & K_{2,3} \\
K_{3,2} & K_{3,3}
\end{array}\right],
$$

respectively.

The Newmark- $\beta$ method [27] is then applied to Eq. (42) to evaluate the acceleration, the velocity and displacement vectors, respectively, at the $n+1$ th calculation step as

$$
\begin{aligned}
& \ddot{\mathbf{q}}^{n+1}=-\tilde{\mathbf{M}}^{-1}\left(\breve{\mathbf{M}} \ddot{\mathbf{q}}^{n}+\breve{\mathbf{C}} \dot{\mathbf{q}}^{n}+\breve{\mathbf{K}} \mathbf{q}^{n}\right) \\
& \dot{\mathbf{q}}^{n+1}=\dot{\mathbf{q}}^{n}+(1-\gamma) h \ddot{\mathbf{q}}^{n}+\gamma h \ddot{\mathbf{q}}^{n+1} \\
& \mathbf{q}^{n+1}=\mathbf{q}^{n}+h \dot{\mathbf{q}}^{n}+(1 / 2-\beta) h^{2} \ddot{\mathbf{q}}^{n}+\beta h^{2} \ddot{\mathbf{q}}^{n+1}
\end{aligned}
$$

where

$$
\tilde{\mathbf{M}}=\mathbf{M}+\mathbf{C} \gamma h+\mathbf{K} \beta h^{2}, \breve{\mathbf{M}}=\mathbf{C}(1-\gamma) h+\mathbf{K}(1 / 2-\beta) h^{2}, \breve{\mathbf{C}}=\mathbf{C}+\mathbf{K} h, \quad \breve{\mathbf{K}}=\mathbf{K}
$$

where $\beta=1 / 6, \gamma=1 / 2$ and $h$ is the time step.

Once the solution of (42) is found, it is then possible to calculate the time history of the generalized coordinates. For a selected detuning parameter $\sigma$, the amplitude $\delta$ of the parametric excitation 
is varied continuously until the time history shifts its behaviour from stable to unstable. The value of $\delta$ leading the system to the instability signals the boundary of the parametric resonance region for the lagging/axial motions of the rotating beam.

\section{Results and discussions}

\subsection{Nonlinear free vibrations analysis}

In this section, numerical simulations aimed at determining the nonlinear free vibrations of the rotating beam are presented. To this aim, an isotropic beam possessing the geometric al and mechanical properties given in Tab. 1 is considered.

Table 1: Geometric al and mechanical properties from Refs. [7] and [16]

\begin{tabular}{cccccc}
\hline $\begin{array}{c}\text { Mass per unit length } \\
(\mathrm{kg} / \mathrm{m})\end{array}$ & $\begin{array}{c}\text { Axial Stiffness } \\
(\mathrm{N})\end{array}$ & $\begin{array}{c}\text { Flexural Stiffness } \\
\left(\mathrm{Nm}^{2}\right)\end{array}$ & $\begin{array}{c}\text { Length } \\
(\mathrm{m})\end{array}$ & $\begin{array}{c}\text { Rotor Radius } \\
(\mathrm{m})\end{array}$ & $\begin{array}{c}\text { Angular Speed } \\
(\mathrm{rad} / \mathrm{s})\end{array}$ \\
\hline 10 & $2.23 \times 10^{8}$ & $3.99 \times 10^{5}$ & 9 & 0.5 & 30 \\
\hline
\end{tabular}

Moreover, further studies are carried out by comparing the results in terms of effective nonlinearity coefficient and principal parametric resonance response so as to illustrate the importance of the exact geometrical formulation (EGF) against ad hoc beam theories such as the Euler-Bernoulli beam model including von-Karman strain-displacement relation (EBVK).

As mentioned above, the mechanical parameters adopted for the case-study beam and reported in Tab. 1 were taken from the literature, although, the mechanical model proposed in [7] and [16] only accounted for the flapping motion of the blade while the lagging motion was neglected. The lowest four lagging natural frequencies and the lowest two axial natural frequencies are compared in Table 2 with those reported in [7].

Table 2: The lagging and axial frequencies ( $\mathrm{rad} / \mathrm{s})$ in comparison with those reported in $[7]$ at rotation speed $\omega_{R}=0$

\begin{tabular}{ccccccc}
\hline Natural frequencies & $1^{\text {st }}$ Lagging & $2^{\text {nd }}$ Lagging & $3^{\text {rd }}$ Lagging & $4^{\text {th }}$ Lagging & $1^{\text {st }}$ Axial & $2^{\text {nd }}$ Axial \\
\hline Ref. [7] results & 8.672 & 54.35 & 152.2 & 298.2 & 824.7 & 2474.0 \\
\hline Current results & 8.671 & 54.330 & 152.071 & 297.825 & 824.195 & 2472.584
\end{tabular}

The results of the asymptotic analysis in terms of effective nonlinearity coefficients for the lowest three lagging modes are validated through a comparison, shown in Tab. (3), with the results reported in [16] for the case of stationary beam. Because of the different nondimensionalization procedure between the current paper and Ref. [16] and the different definition of the effective nonlinearity 
coefficient, to compare the analogous quantities the current results must be re-calculated as $\Gamma_{k, k}=$ $4 \omega_{0} \gamma_{k}$.

After validation of the current results, a comprehensive study on the effects of the angular speed on the effective nonlinearity coefficients is investigated next. The considered beam [15] is isotropic and has a rectangular cross-section. The rotor radius, the beam width, the beam thickness and the beam length are $0.2 \mathrm{~m}, 0.05 \mathrm{~m}, 0.005 \mathrm{~m}$, and $2 \mathrm{~m}$, respectively. The mechanical properties are $E=70 \mathrm{GPa}, G=26 \mathrm{GPa}$, and the mass density is $\rho=2700 \mathrm{~kg} / \mathrm{m}^{3}$. The variation with the of the lowest natural frequency of the lagging and axial modes are depicted in Fig. 2. This figure shows the $1: 1$ and $2: 1$ internal resonance possibilities between the lagging and axial modes.

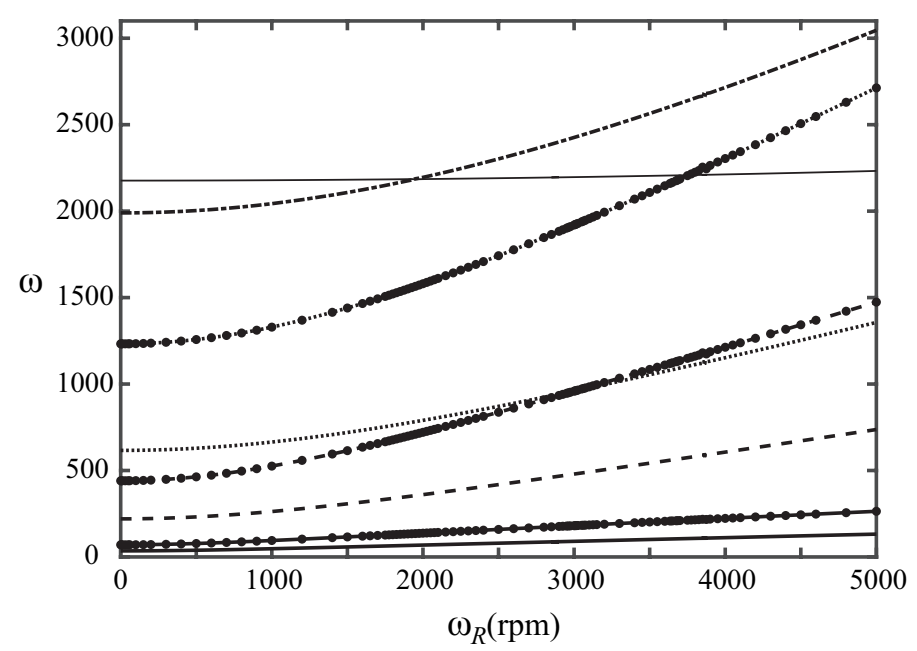

Figure 2: Frequency loci of the lagging and axial modes: first axial mode (thin line), first lagging mode (thick line), second lagging mode (dashed-line), third lagging mode (dotted-line), and fifth lagging mode (dotted-dashed-line). Filled circles indicate the modes having frequency twice the corresponding mode.

It is evident that a $1: 1$ internal resonance can exist at a rotational speed of $1940 \mathrm{rpm}$ between the first axial mode and the fifth lagging mode. On the other hand, two $2: 1$ internal resonances between the second and third lagging modes at a rotational speed of $3016 \mathrm{rpm}$ and between the third lagging mode and the first axial mode at $3757 \mathrm{rpm}$ also exist.

Table 3: The effective nonlinearity coefficient, $\Gamma_{k, k}=4 \omega_{0} \gamma_{k}$, for the first three lagging modes in comparison with those of Ref. [16] at rotation speed $\omega_{R}=0$

\begin{tabular}{cccc}
\hline & $\Gamma_{1,1}$ & $\Gamma_{2,2}$ & $\Gamma_{3,3}$ \\
\hline Ref. [16] results & -619690 & 18548339 & 63563664 \\
\hline Current results & -630380 & 18218000 & 61543000 \\
\hline Difference error percent & 1.72 & 1.78 & 3.18
\end{tabular}


The corresponding effective nonlinearity coefficient $\gamma_{1}$ for the first lagging mode is depicted in Fig. 3-(a). In particular, solid lines refer to the results obtained using the EGF model, while filled circles indicate the results of the EBVK models. It is clear that for a stationary beam, i.e., when $\omega_{R}=0$, a softening behaviour is predicted for the first lagging mode. It is worth to note that the results obtained by means of the EGF model show that the rotating beam starts with a sharp softening nonlinearity and then it moves to a hardening nonlinearity, conversely, the EBVK model predicts invariant softening nonlinearity. On the other hand, for higher rotational speed the difference between the results obtained via EGF and EBVK models increases considerably.

The effective nonlinearity coefficient $\gamma_{2}$ of the second lagging mode is shown in Fig. 3-(b). A hardening nonlinearity is predicted for the second lagging mode of the stationary beam. As mentioned before, 2: 1 internal resonance occurs between the second and the third lagging modes at $3016 \mathrm{rpm}$ causing a singularity in the solution. It is obvious that the nonlinearity type does not change around the singularity and, therefore, a hardening type of nonlinearity is predicted for this mode all over the investigated range of angular speeds.

Finally, the effective nonlinearity coefficient $\gamma_{3}$ of the third lagging mode is presented in Fig. 3-(c). The third lagging mode displays a hardening treatment for the stationary beams. The $2: 1$ internal resonances between the third lagging and first axial mode at $3757 \mathrm{rpm}$ is evident. However, a change in the nonlinearity type around the singularity point is predicted for this mode. Except this change in the nonlinearity type around this speed, the beam shows a hardening type of nonlinearity at whole of the rotation speed range.

The comparative analysis performed to highlight the difference between the results of the here proposed EGF model and the EBVK model, showed that the two modeling approach deliver results which differ drastically for higher rotational speeds. This discrepancy is more evident for the first and the second lagging modes, see Tab. 4. Moreover, although the comparison was performed for symmetric beams, the discrepancy found between the aforementioned theories is expected to grow for non-symmetric beams since the lagging deformation arises in the prestressed configuration and at moderate-to-high speeds, the chord-wise deformation gets larger. Within this context, the Euler-Bernoulli beam model cannot predict the correct deformation. Consequently, the linear free vibration features and the nonlinear properties suffer further discrepancies.

The nonlinear dynamic response of the lowest two axial modes is studied next. The effective nonlinearity coefficient of the first axial mode, i.e., $\gamma_{1}$, is shown in Fig. $4-(a)$. The figure shows that stationary beams do not show any nonlinearity for the axial modes. As a result of $1: 1$ internal resonances between the first axial mode and fifth lagging mode, a singularity occurs at $1940 \mathrm{rpm}$. Although for stationary beams none nonlinear behavior is predicted, on the other hand, for rotating beams a hardening type of nonlinearity is evident and the singularity does not change the type of nonlinearity. 

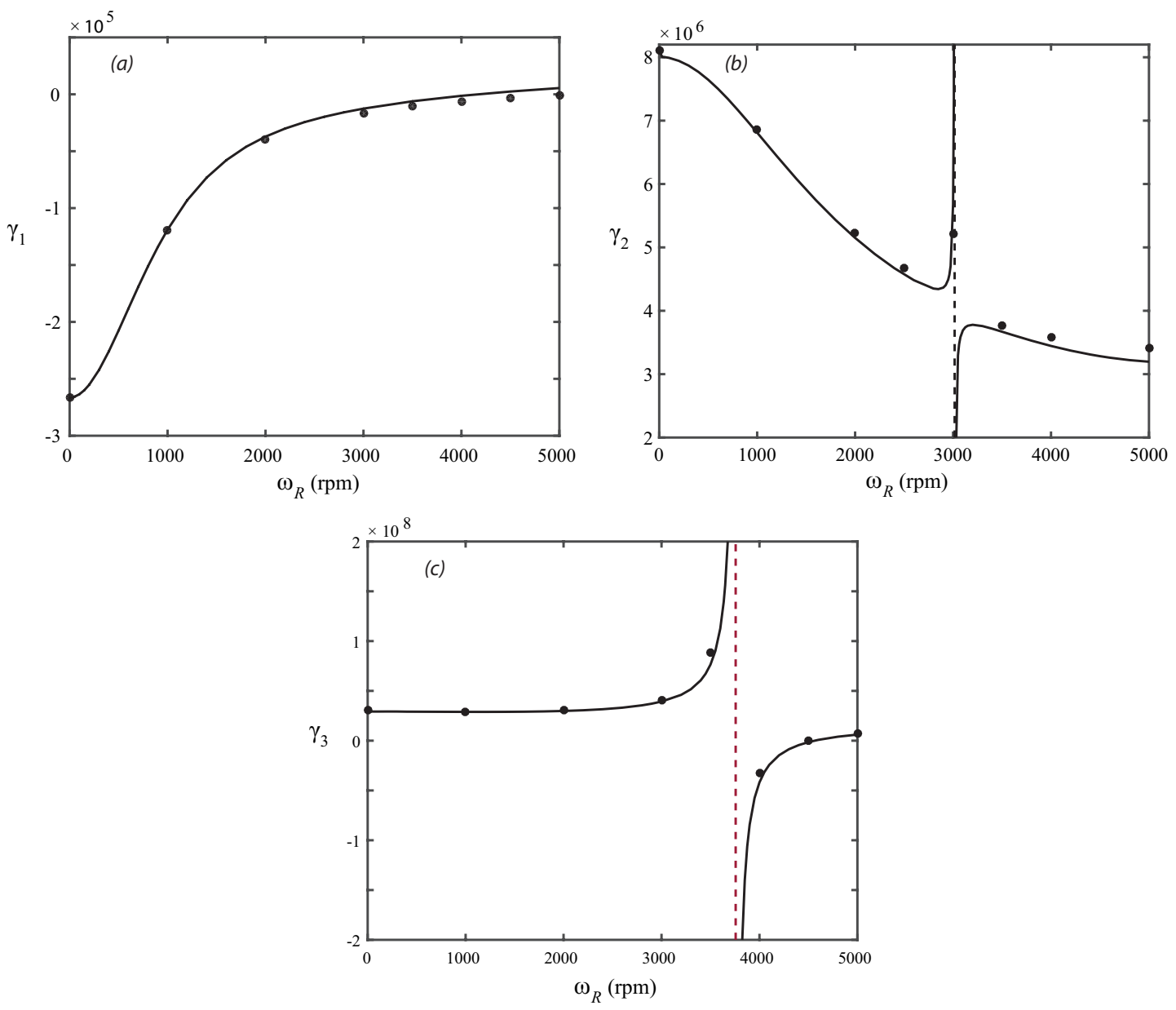

Figure 3: The effective nonlinearity for $(a)$ - the first, $(b)$ - the second and $(c)$ - the third lagging modes: On the basis of the exact geometrical formulation (solid-lines) vs. those of Euler-Bernoulli beam theory (filled circles).

The variation of the effective nonlinearity coefficient $\gamma_{2}$ of the second axial mode is illustrated in Fig. 4-(b). The same qualitative results get for the first axial mode are also obtained for the second. However, no singularity arises for this mode.

Table 4: The prediction of the first three lagging effective nonlinearities based on the EGF model versus the EBVK model

\begin{tabular}{cccccccccc}
\hline & \multicolumn{3}{c}{ EGF } & & \multicolumn{2}{c}{ EBVK } & \multicolumn{3}{c}{ Difference \% } \\
\hline & $0 \mathrm{rpm}$ & $3000 \mathrm{rpm}$ & $5000 \mathrm{rpm}$ & $0 \mathrm{rpm}$ & $3000 \mathrm{rpm}$ & $5000 \mathrm{rpm}$ & $0 \mathrm{rpm}$ & $3000 \mathrm{rpm}$ & $5000 \mathrm{rpm}$ \\
\hline$\gamma_{1}$ & -266891.0 & -12577.7 & 5493.0 & -266724.2 & -16464.7 & -1069.7 & 0.1 & 30.9 & 119.5 \\
\hline$\gamma_{2}$ & 8072985.7 & 5680813.0 & 3195947.1 & 8105767.6 & 5215420.7 & 3418463.5 & 0.4 & 8.2 & 7.0 \\
\hline$\gamma_{3}$ & 30554094.5 & 39279895.7 & 6103294.1 & 30828726.8 & 40820985.2 & 7059566.5 & 0.9 & 3.9 & 15.7 \\
\hline
\end{tabular}



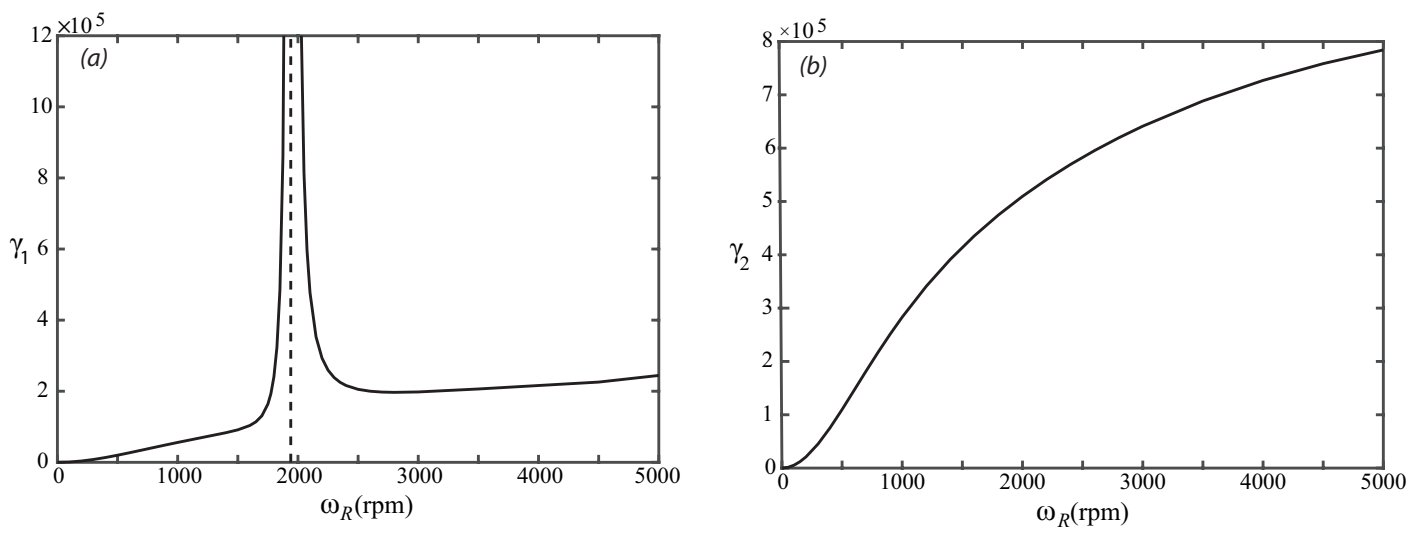

Figure 4: The effective nonlinearity for $(a)$ - the first and $(b)$ - the second axial modes.

\subsection{Principal parametric resonance analysis}

To show the validity of the proposed solution method in the prediction of the dynamic response of the rotating beam undergoing principal parametric resonance, some numerical results are shown and compared with those obtained in [21]. The parametric excitation frequency is assumed to be twice the axial frequency, i.e., $\Omega=2 \omega_{3, k}+\varepsilon^{2} \sigma$. According to the data reported in [21], the beam rotates at $1500 \mathrm{rpm}$, is made of Aluminium, and the geometric and mechanical parameters are: $E=70(G P a), G=26(G P a), \rho=2700, d_{3}=0.5(m), L=5(m), b=0.05(m)$ and $h=0.005(m)$. The stability regions of the principal parametric resonance are calculated for the first and second axial modes, respectively, and are shown in Fig. 5 where a comparison with the results obtained in [21] is also proposed. It is worth noting that, although in [21] the coupling of the lagging-axial motions was not considered, nevertheless, the results obtained in this work show the reliability of the here proposed method.
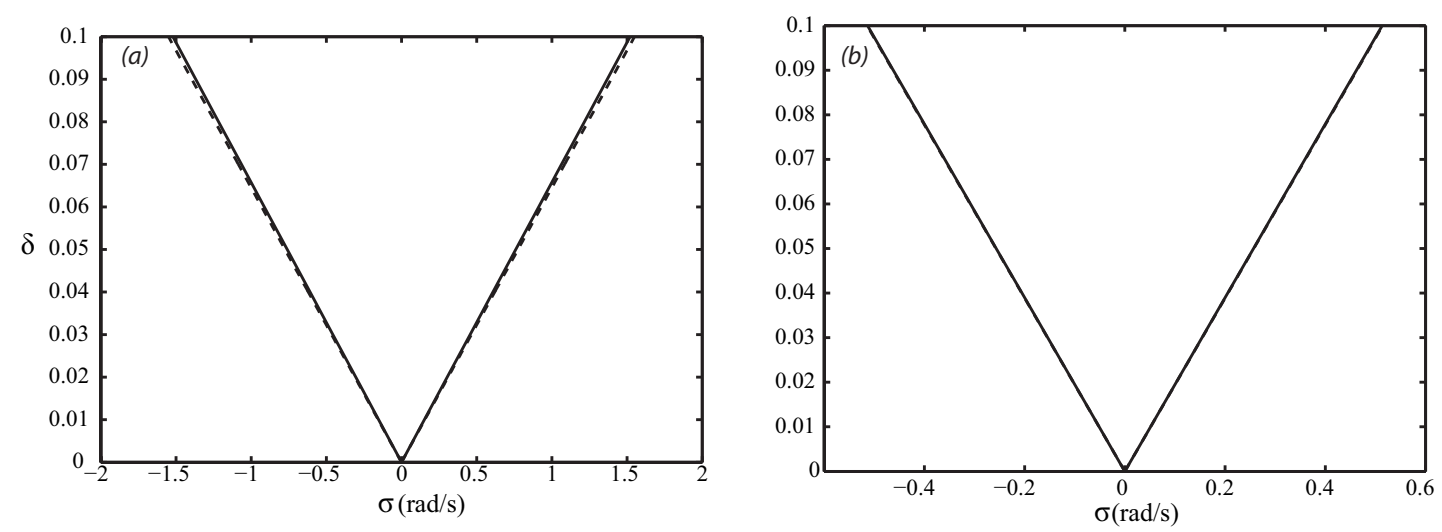

Figure 5: The principal parametric resonance stability region boundaries for $(a)$ - the first and $(b)$ - the second, axial modes: Current MMS results (solid-lines) vs. those of Ref. [21] (dashed-lines). 
The MMS vs. the Newmark approach. Further validation of the semi-analytical results obtained via MMS in the study of the parametric resonance of rotating beams is proposed by implementing Newmark method to solve numerically the equations of motion. Henceforth, the mechanical parameters presented in the previous section are considered. The beam rotational speed is $1000 \mathrm{rpm}$. In this case, the parametric excitation frequency is assumed to be $\Omega=2 \omega_{2,1}+\varepsilon \sigma$ and the stability region is depicted in Fig. 6. The results demonstrated the validity of the current MMS. The discrepancy between the results is due to the more precise results of numerical techniques. Although the numerical outcome is more precise, for all points in this diagram numerous iterations are required i.e. the time history obtained by the Newmark method for any specific value of detuning parameter is analyzed by perturbing the parametric excitation amplitude $\delta$ until the time history response becomes unstable and the value of $\delta$ is stored as the threshold value for the instability region. The operation is then repeated in the selected range of detuning parameter $\sigma$.

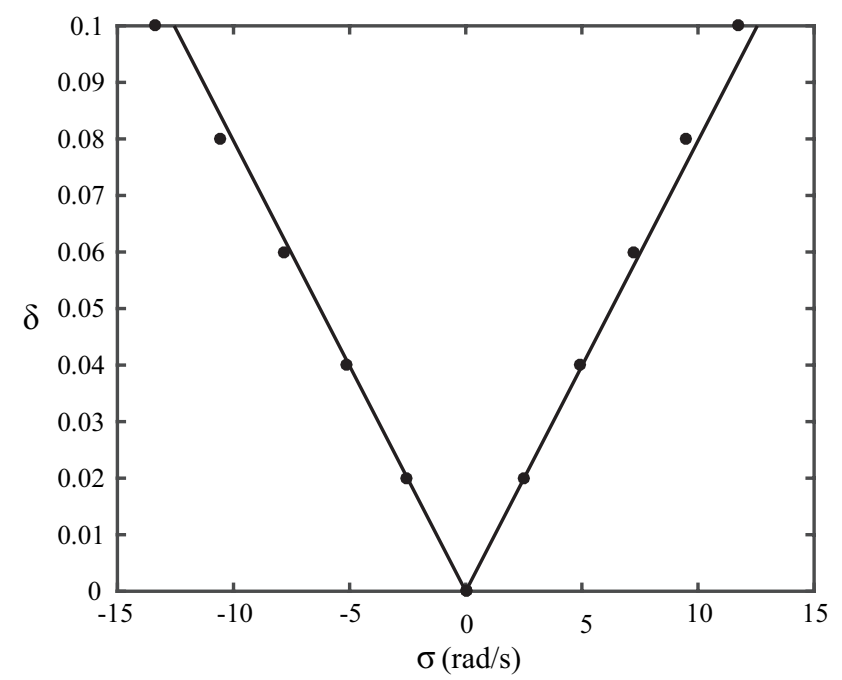

Figure 6: The principal parametric resonance stability region boundaries for the first lagging mode: Current MMS results (solid-lines) vs. those of Newmark method (filled circles).

After validation of the MMS results, principal parametric resonance for the lagging-axial motions is investigated next.

The effect of the structural damping for three different values of the damping ratio for the first lagging mode and the first axial mode is shown in Fig. 7. In the simulations performed $\zeta_{2}=\zeta_{3}$. The results show that, as expected, by increasing the damping ratio the minimum value of the excitation amplitude $\delta$, necessary to activate the parametric resonance, increases with the damping.

The effect of the angular speed in the stability region of the principal parametric resonance for the first lagging and axial modes is shown in Fig. 8. It can be seen that, by increasing the rotating speed the instability region is broadening. 

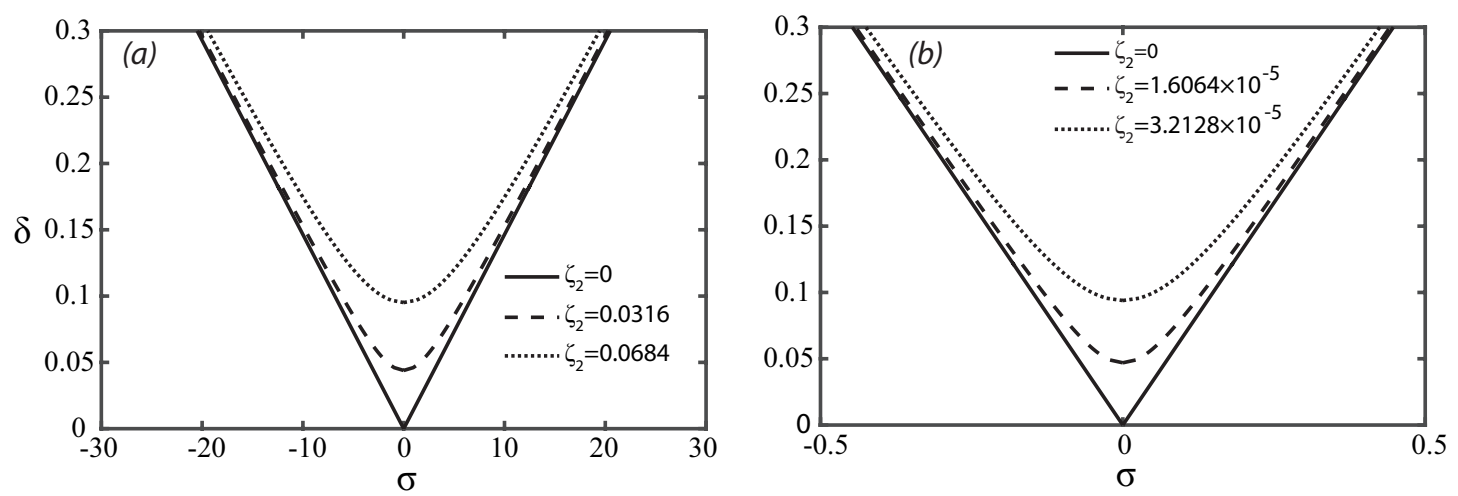

Figure 7: The principal parametric resonance stability region boundaries for the $(a)$ - first lagging modes: $\zeta_{2}=0$ (solid-lines), $\zeta_{2}=0.0316$ (dashed-lines) and $\zeta_{2}=0.0684$ (dotted-lines) and (b)- first axial mode: $\zeta_{2}=0$ (solid-lines), $\zeta_{2}=1.6064 \times 10^{-5}$ (dashed-lines) and $\zeta_{2}=3.2128 \times 10^{-5}$ (dotted-lines).
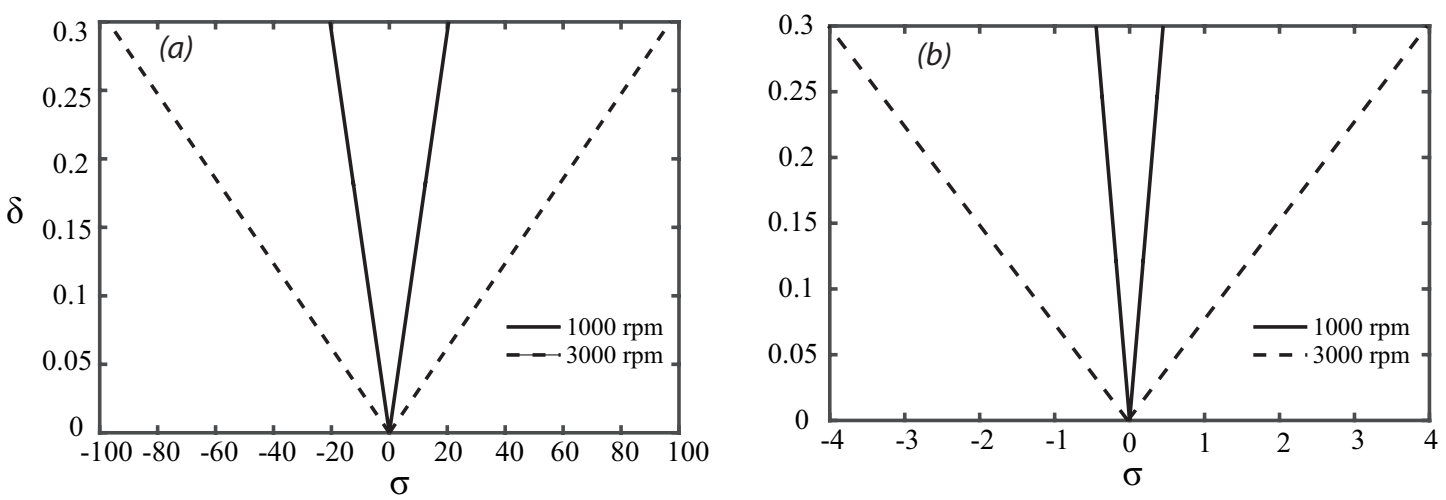

Figure 8: The principal parametric resonance stability region boundaries for the first (a)-lagging and (b)-axial modes: 1000rpm (solid-lines) vs. 3000rpm (dashed-lines).

The stability regions for the first and second lagging modes calculated by adopting the EGF model and EBVK model, respectively, are depicted in Fig. 9 for two different rotational speeds. It can be seen that, in agreement with Tab. 4, the role of the implemented model is more highlighted in determining the first lagging mode instability region boundary rather than the second one especially at high speeds. Moreover, the model based on the exact geometrical formulation determines a wider instability region comparing with the Euler-Bernoulli beam model.

Sensitivity to rotational speed. The instability region boundary sensitivity to the rotation speed is studied next. In this study, the rotational speed is varied from 0 to $5000 \mathrm{rpm}$ and the parametric excitation amplitude $\delta$ is set to 0.1 . Beside the variation of the natural frequencies in each rotating speed, the detuning parameter is also varying. The parametric excitation frequency is set to be 

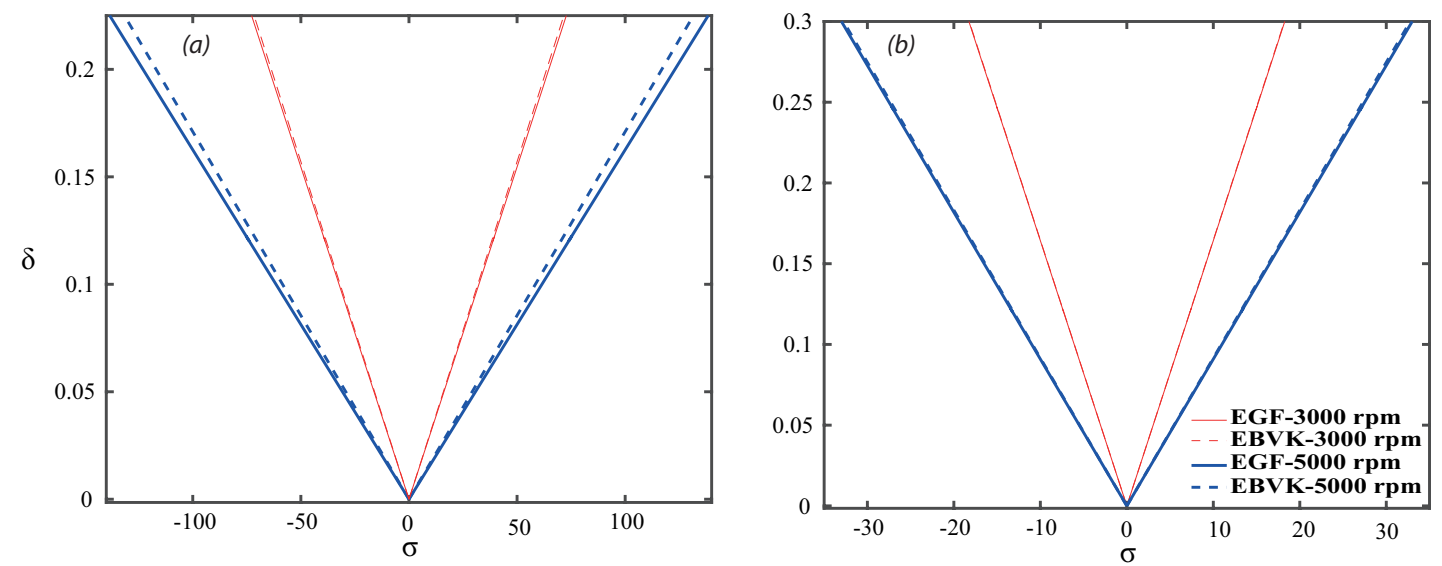

Figure 9: The principal parametric resonance stability region boundaries for the $(a)$-first and $(b)$-second lagging modes: 3000rpm (thin-lines) vs. 5000rpm (thick-lines).

$\Omega=2 \omega_{k}+\sigma$ for a selected rotational speed.

The boundaries of the instability region for the lowest three lagging modes is shown in Fig. 10 in terms of the rotational speed. In the abscissa is reported the nondimensional parametric excitation frequency $\Omega$. The figure shows that, by increasing the rotational speed, the instability region of the parametric resonance becomes wider. On the other hand, the instability region of the lowest modes is broader than that of the higher modes.
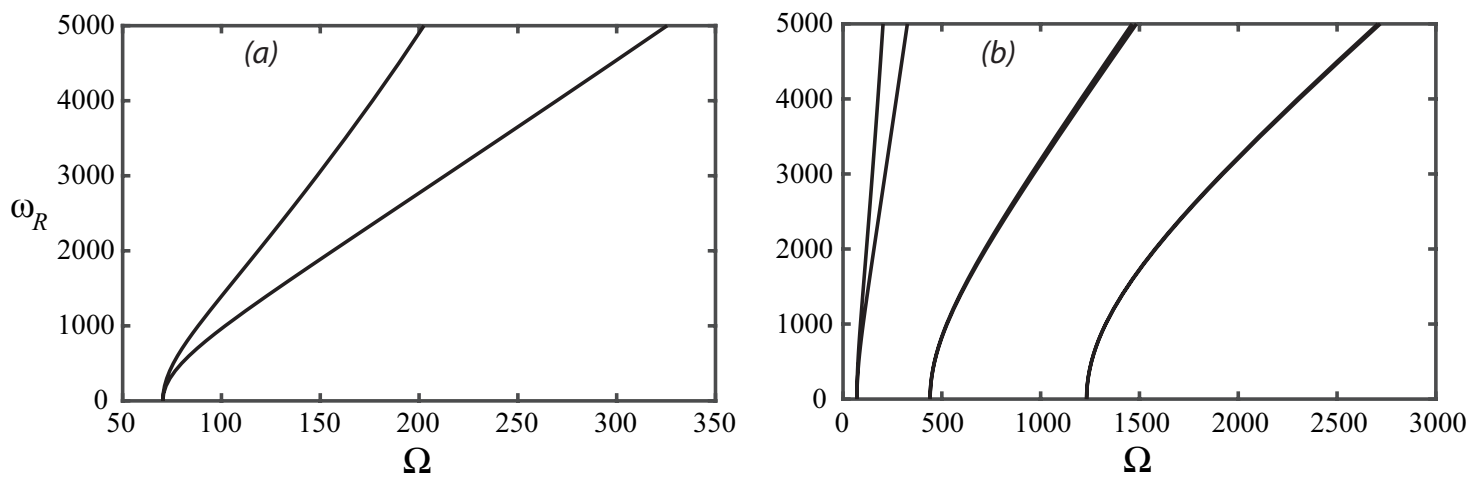

Figure 10: The principal parametric resonance stability region boundaries for the $(a)$ - first and $(b)$ - first three lagging modes in terms of the rotating speed and the dimensionless parametric excitation frequency.

A similar analysis has been conducted for the lowest two axial modes and the results presented in Fig. 11.

Post-critical response. The post-critical response of the lagging-axial modes undergoing parametric response is examined in this section for $\delta=0.1$. The influence of the structural damping on 

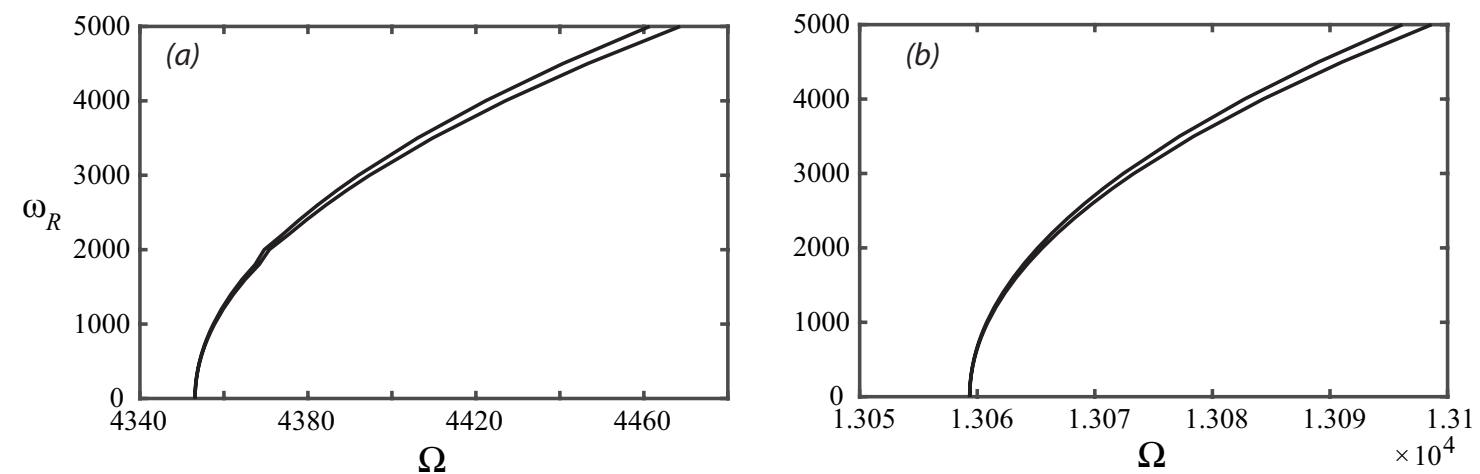

Figure 11: The principal parametric resonance stability region boundaries for the $(a)$ - first and $(b)$ - second axial modes in terms of the rotating speed and the dimensionless parametric excitation frequency.

the parametric resonance of the first lagging mode and first axial mode, respectively, is shown in Fig. 12 for a rotational speed of $1000 \mathrm{rpm}$. By varying the detuning parameter from left to right a subcritical pitchfork bifurcation occurs for the first lagging mode which the stable trivial solution coexists with an unstable periodic solution (see Fig. 12 (a)). Thereafter, by increasing the detuning parameter $\sigma$, the subcritical pitchfork bifurcation is followed by a supercritical pitchfork bifurcation and so the stable trivial solution turns into an unstable solution while another periodic solution is born which is stable and attracts the beam dynamics. On the other hand, Fig. 12 (b) shows an hardening behaviour of the first axial mode. The softening and hardening behaviours of the studied modes are in agreement with the presented results in the previous section.
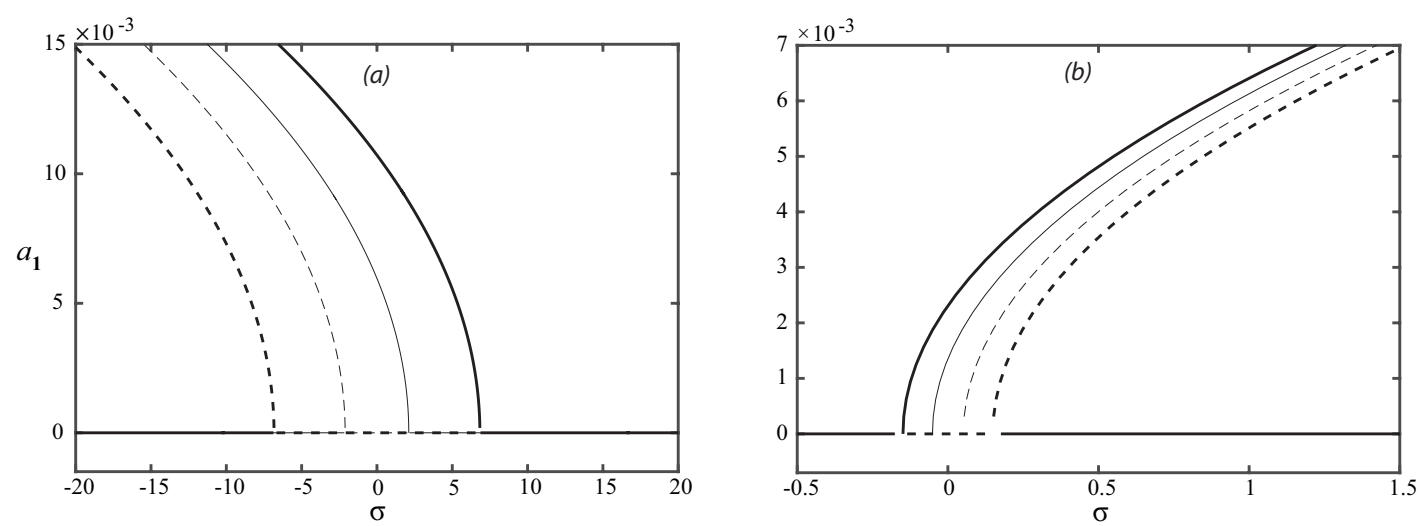

Figure 12: The parametric resonance response for the first $(a)$ - lagging mode: $\zeta_{2}=0$ (thick-lines) and $\zeta_{2}=0.0684$ (thin-lines) and (b)- axial mode: $\zeta_{2}=0$ (thick-lines) and $\zeta_{2}=3.2128 \times 10^{-5}$ (thin-lines).

The influence of the rotational speed in the parametric resonance of the lowest three lagging modes and two axial modes, respectively, are depicted in Figs. 13 and 14. It is clear that the instability region is wider for the higher rotational speeds. On the other hand, according to the results in terms of the effective nonlinearity coefficients presented in the previous section, the type 
of nonlinearity is invariant for the lowest three lagging modes and two axial modes when the beam rotates at $1000 \mathrm{rpm}$ and $3000 \mathrm{rpm}$, respectively.
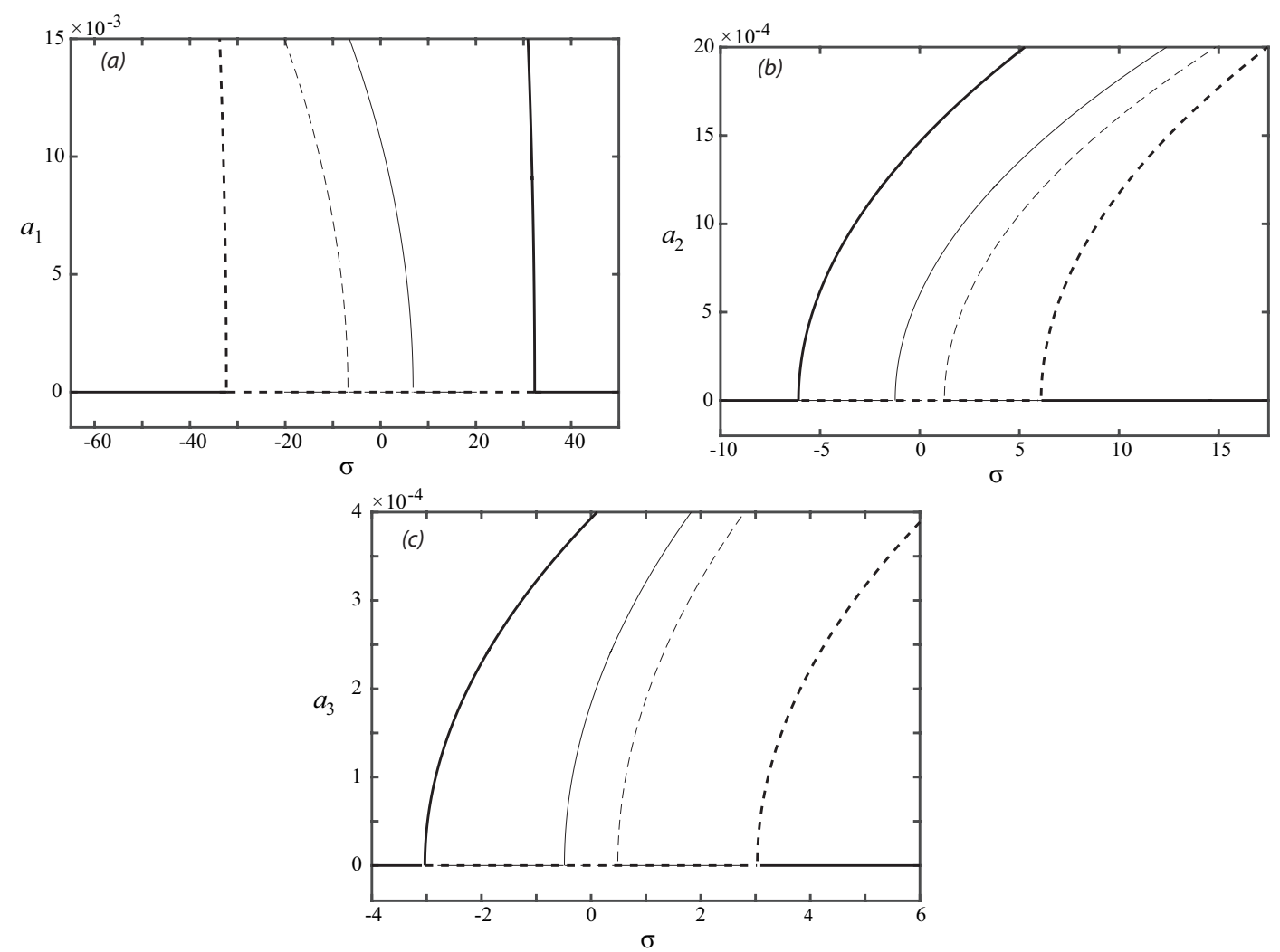

Figure 13: Parametric resonance response for the $(a)$-first, $(b)$-second and $(c)$-third lagging modes: 1000rpm (thin-lines) vs. 3000rpm (thick-lines).

The importance of the employed beam theory on the principal parametric resonance response for the first and second lagging modes is shown in Fig. 15. It is to be noted that only the right branch of the response is shown. The results highlight the importance of the implemented theory with respect to rotation speed increments especially for the first lagging mode. Moreover, a qualitative difference is also observed in the principal parametric resonance treatment of the first lagging mode at $5000 \mathrm{rpm}$. In other words, although the result on the basis of the EGF predicts a stable trivial solution with one stable and one unstable periodic solutions with the increase of detuning parameter, considering the whole treatment including the left and the right branches in mind, the EBVK predicts a softening nonlinearity. Accordingly, the latter anticipates one stable trivial solution with the increase of detuning parameter. 

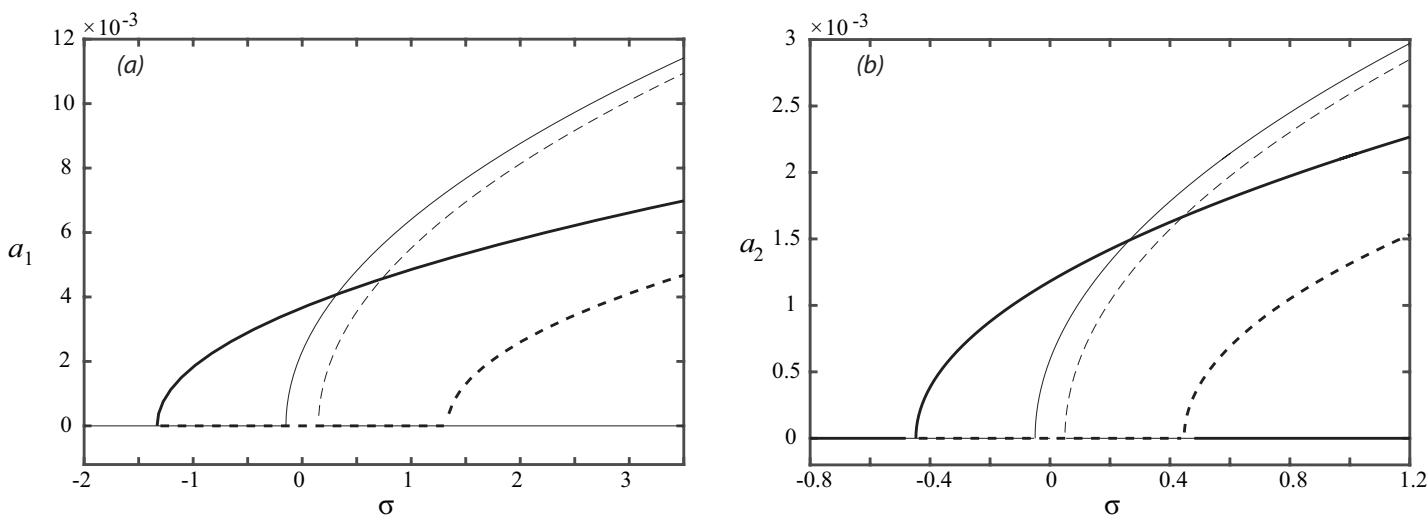

Figure 14: Parametric resonance response for the (a)-first and (b)-second axial modes: 1000rpm (thin-lines) vs. $3000 \mathrm{rpm}$ (thick-lines).
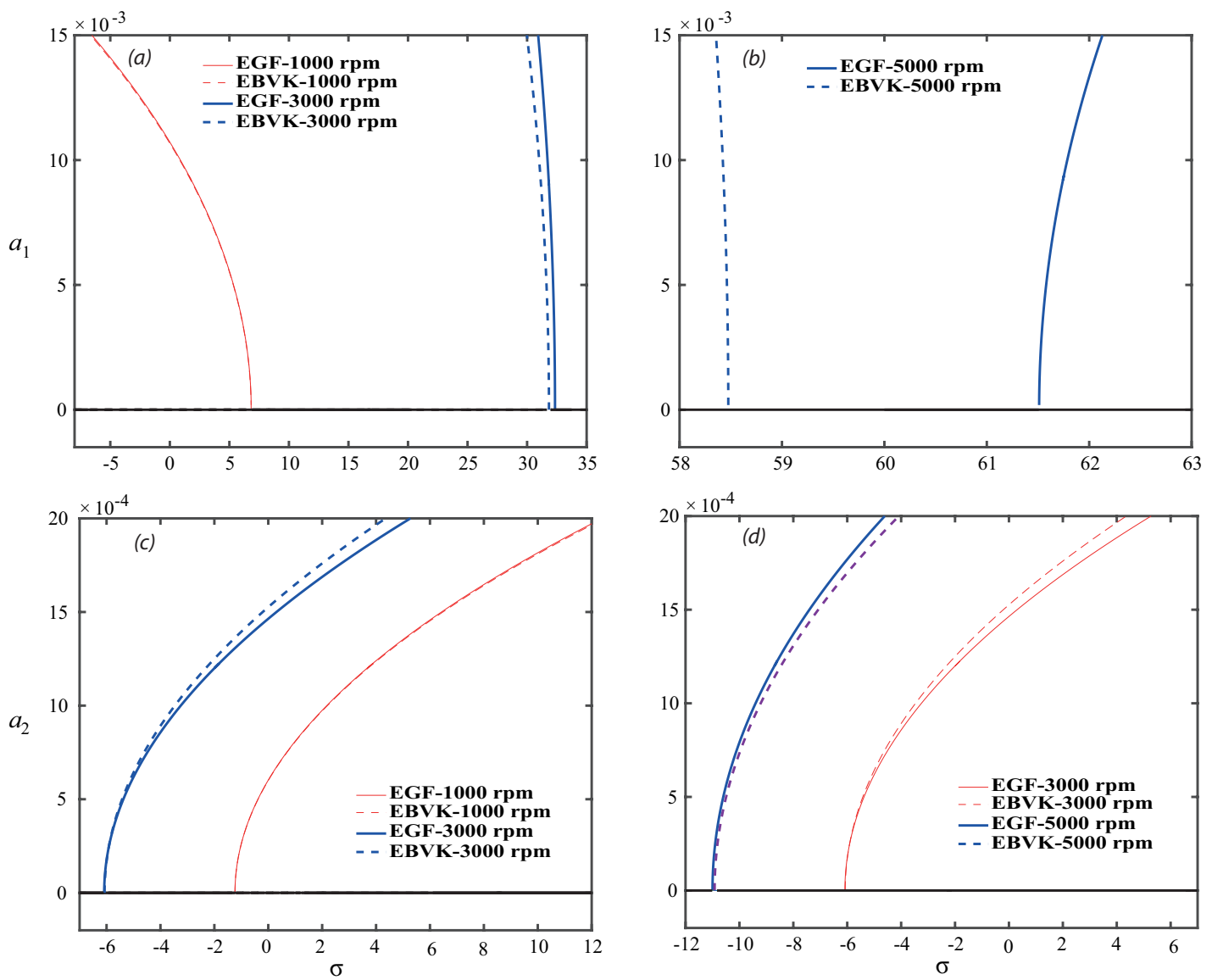

Figure 15: Parametric resonance response for the first lagging mode: (a)- 1000rpm (thin-lines) vs. $3000 \mathrm{rpm}$ (thick-lines) and (b)- 5000rpm and the second lagging mode: (c)-1000rpm (thin-lines) vs. 3000rpm (thicklines) and (d)-3000rpm (thin-lines) vs. 5000rpm (thick-lines). (EGF-based on the exact geometrical formulation; EBVK-based on the Euler-Bernoulli beam assumption.) 


\section{Conclusions}

In this paper, lagging-axial nonlinear free vibrations and principal parametric resonances are examined in the presence of Coriolis forces in the context of a geometrically exact rotating beam formulation. The direct method of multiple scale was implemented to deliver the effective nonlinearity coefficients of various modes, the transition curves (i.e., the parametric instability regions boundaries) and the parametric resonance motion treatment. Comparisons in terms of effective nonlinearity coefficient and principal parametric resonance response were carried out so as to illustrate the importance of the exact geometrical formulation against ad hoc beam theories such as the Euler-Bernoulli beam model. A comprehensive study was carried out to show the change in nonlinearity suffered by lagging-axial modes upon angular speed variations. In addition, the nonlinear parametric resonance behaviour and the stability region boundaries were examined in terms of the rotation speed and the damping ratio. In the context of nonlinear free vibrations, the results led to the following highlights: first, the axial modes do not show any type of nonlinearity for the stationary beams but, for the rotating beams due to the Coriolis forces induced coupling, a hardening nonlinearity is manifested. Second, $1: 1$ internal resonances between the lagging and axial modes and $2: 1$ internal resonances between the lagging modes do not change the type of nonlinearity around the singularity caused in the effective nonlinearity coefficient. However, but $2: 1$ resonances between the lagging and axial modes can shift the type of nonlinearity around it. Third, a softening-hardening nonlinearity is predicted for the first lagging mode while a hardening nonlinearity is computed for the second and third lagging modes (except in the vicinity of the singularity). Moreover, the EulerBernoulli beam model fails to predict the precise effective nonlinearity especially for the first lagging mode for moderate-to-high rotating speeds and for some rotation speeds the softening/hardening type of nonlinearity was predicted erroneously.

On the other hand, in the context of the parametric resonances, the main findings can be summarized as follows. (i) When the lagging and axial modes undergo parametric resonances, the range of the associated instability regions, in terms of detuning parameter, decreases with the mode number. (ii) The instability region when the lagging modes are excited is more flattened than that of the axial modes. (iii) The study into the full onset of parametric resonance motions reveals that when the lagging and axial modes are subject to a parametric resonance excitation the loss of stability of the trivial solution gives rise to the onset of period doubled oscillations governed by a nonlinear frequency response exhibiting the same type of nonlinearity of the involved mode. (iv) The difference between the results predicted in terms of the implemented beam theory is manifested by the increment of the rotation speed and is higher for the first lagging mode. Also, the instability region predicted resorting the Euler-Bernoulli beam assumption is narrower than the associated one defined by the exact geometrical formulation. In addition, a qualitatively wrong parametric resonance response is determined by the Euler-Bernoulli beam model. 
Appendix I: The linear inertia, gyroscopic and stiffness operators

$$
\begin{aligned}
I_{22} & =-(\bullet)+\nu_{i n v}^{0}(s)^{2} \partial_{s s}(\bullet) / \alpha_{12}+2 \nu_{i n v}^{0}{ }^{\prime}(s) \partial_{s}(\bullet) \nu_{i n v}^{0}(s) / \alpha_{12}, \quad I_{23}=0, \quad G_{22}=0, \quad G_{23}=2 \lambda(\bullet) \\
L_{22} & =\lambda^{2}(\bullet)-\alpha_{22} \partial_{s}(\bullet) \nu_{i n v}^{0}{ }^{\prime \prime \prime}(s) \nu_{i n v}^{0}(s) / \alpha_{12}+\alpha_{22} \partial_{s s}(\bullet) N^{0}(s) \nu_{i n v}^{0}(s)-2 \alpha_{22} \nu_{i n v}^{0}{ }^{\prime}(s)^{2} \partial_{s s}(\bullet) / \alpha_{12} \\
& -4 \alpha_{22} \nu_{i n v}^{0}{ }^{\prime}(s) \partial_{s s s}(\bullet) \nu_{i n v}^{0}(s) / \alpha_{12}+\alpha_{22} \partial_{s}(\bullet) N^{0 \prime}(s) \nu_{i n v}^{0}(s)+\alpha_{22} \partial_{s}(\bullet) N^{0}(s) \nu_{i n v}^{0}{ }^{\prime}(s) \\
& -\alpha_{22} \nu_{i n v}^{0}{ }^{\prime}(s) \partial_{s}(\bullet) \nu_{i n v}^{0}{ }^{\prime \prime}(s) / \alpha_{12}-3 \alpha_{22} \partial_{s s}(\bullet) \nu_{i n v}^{0}{ }^{\prime \prime}(s) \nu_{i n v}^{0}(s) / \alpha_{12}-\alpha_{22} \nu_{i n v}^{0}(s)^{2} \partial_{s s s s}(\bullet) / \alpha_{12}, \quad L_{23}=\dot{\lambda}(\bullet)
\end{aligned}
$$

$$
I_{33}=-(\bullet), \quad I_{32}=0, \quad G_{32}=-2 \lambda(\bullet), \quad G_{33}=0, \quad L_{32}=-\dot{\lambda}(\bullet), \quad L_{33}=\alpha_{22} \partial_{s s}(\bullet)+\lambda^{2}(\bullet)
$$

$$
\begin{aligned}
& I_{B C 21}=\partial_{s}(\bullet) / \alpha_{12}, \quad L_{B C 21}=-\alpha_{22} \partial_{s s s}(\bullet) / \alpha_{12}-\alpha_{22} \partial_{s}(\bullet) \nu_{i n v}^{0}{ }^{\prime \prime} / \alpha_{12}-2 \alpha_{22} \partial_{s s}(\bullet) \nu_{i n v}^{0}{ }^{\prime} / \alpha_{12} \\
& L_{B C 22}=-\alpha_{22} \partial_{s}(\bullet) \nu_{i n v}^{0}{ }^{\prime} / \alpha_{12}-\alpha_{22} \partial_{s s}(\bullet) / \alpha_{12} \\
& L_{B C 3}=\alpha_{22} \partial_{s}(\bullet)
\end{aligned}
$$

\section{Appendix II: Quadratic inertia and stiffness operators}

$$
i_{21}^{(2)}=-2 \nu_{i n v}^{0}(s)^{3} \partial_{t s} u_{2} \partial_{t s s} u_{3} / \alpha_{12}-2 \nu_{i n v}^{0}(s)^{3} \partial_{t s s} u_{2} \partial_{t s} u_{3} / \alpha_{12}-6 \nu_{i n v}^{0}(s)^{2} \nu_{i n v}^{0}{ }^{\prime}(s) \partial_{t s} u_{2} \partial_{t s} u_{3} / \alpha_{12}
$$

$$
\begin{aligned}
i_{22}^{(2)} & =-2 \nu_{i n v}^{0}(s)^{3} \partial_{s} u_{3} \partial_{t t s s} u_{2} / \alpha_{12}-\nu_{i n v}^{0}(s)^{3} \partial_{s s} u_{2} \partial_{t t s} u_{3} / \alpha_{12}-\nu_{i n v}^{0}(s)^{3} \partial_{s} u_{2} \partial_{t t s s} u_{3} / \alpha_{12} \\
& -2 \nu_{i n v}^{0}(s)^{3} \partial_{s s} u_{3} \partial_{t t s} u_{2} / \alpha_{12}-6 \nu_{i n v}^{0}(s)^{2} \partial_{s} u_{3} \nu_{i n v}^{0}{ }^{\prime}(s) \partial_{t t s} u_{2} / \alpha_{12}-3 \nu_{i n v}^{0}(s)^{2} \nu_{i n v}^{0}{ }^{\prime}(s) \partial_{s} u_{2} \partial_{t t s} u_{3} / \alpha_{12}
\end{aligned}
$$

$$
\begin{aligned}
n_{2}^{(2)} & =3 \alpha_{22} \nu_{i n v}^{0}(s)^{2} \partial_{s} u_{3} \partial_{s} u_{2} \nu_{i n v}^{0}{ }^{\prime \prime \prime}(s) / \alpha_{12}+4 \alpha_{22} \nu_{i n v}^{0}(s)^{3} \partial_{s s} u_{3} \partial_{s s s} u_{2} / \alpha_{12} \\
& -\alpha_{22} \nu_{i n v}^{0}(s)^{2} \partial_{s} u_{2} N^{0}(s) \partial_{s s} u_{3}+\alpha_{22} \nu_{i n v}^{0}(s)^{3} \partial_{s} u_{2} \partial_{s s s s} u_{3} / \alpha_{12}+\alpha_{22} \nu_{i n v}^{0}(s) \partial_{s} u_{3} \partial_{s s} u_{2} \\
& +\alpha_{22} \nu_{i n v}^{0}(s) \partial_{s} u_{2} \partial_{s s} u_{3}+2 \alpha_{22} \nu_{i n v}^{0}(s)^{3} \partial_{s} u_{3} \partial_{s s s s} u_{2} / \alpha_{12}+\alpha_{22} \partial_{s} u_{3} \partial_{s} u_{2} \nu_{i n v}^{0}{ }^{\prime}(s) \\
& -\alpha_{22} \nu_{i n v}^{0}(s)^{2} N^{0 \prime}(s) \partial_{s} u_{3} \partial_{s} u_{2}+2 \alpha_{22} \nu_{i n v}^{0}{ }^{\prime}(s)^{3} \partial_{s} u_{2} \partial_{s} u_{3} / \alpha_{12}+3 \alpha_{22} \nu_{i n v}^{0}(s)^{3} \partial_{s s} u_{2} \partial_{s s s} u_{3} / \alpha_{12} \\
& +10 \alpha_{22} \nu_{i n v}^{0}{ }^{\prime}(s) \partial_{s} u_{2} \partial_{s} u_{3} \nu_{i n v}^{0}{ }^{\prime \prime}(s) \nu_{i n v}^{0}(s) / \alpha_{12}+7 \alpha_{22} \nu_{i n v}^{0}(s)^{2} \partial_{s s} u_{3} \partial_{s} u_{2} \nu_{i n v}^{0}{ }^{\prime \prime}(s) / \alpha_{12} \\
& +7 \alpha_{22} \nu_{i n v}^{0}(s)^{2} \nu_{i n v}^{0}{ }^{\prime}(s) \partial_{s} u_{2} \partial_{s s s} u_{3} / \alpha_{12}+12 \alpha_{22} \nu_{i n v}^{0}(s)^{2} \partial_{s} u_{3} \nu_{i n v}^{0}{ }^{\prime}(s) \partial_{s s s} u_{2} / \alpha_{12} \\
& +10 \alpha_{22} \nu_{i n v}^{0}{ }^{\prime}(s)^{2} \partial_{s} u_{2} \partial_{s s} u_{3} \nu_{i n v}^{0}(s) / \alpha_{12}+16 \alpha_{22} \nu_{i n v}^{0}(s)^{2} \partial_{s s} u_{3} \partial_{s s} u_{2} \nu_{i n v}^{0}{ }^{\prime}(s) / \alpha_{12} \\
& +14 \alpha_{22} \nu_{i n v}^{0}{ }^{\prime}(s)^{2} \partial_{s s} u_{2} \partial_{s} u_{3} \nu_{i n v}^{0}(s) / \alpha_{12}+9 \alpha_{22} \nu_{i n v}^{0}(s)^{2} \partial_{s} u_{3} \partial_{s s} u_{2} \nu_{i n v}^{0}{ }^{\prime \prime}(s) / \alpha_{12} \\
& -2 \alpha_{22} \partial_{s} u_{2} N^{0}(s) \partial_{s} u_{3} \nu_{i n v}^{0}{ }^{\prime}(s) \nu_{i n v}^{0}(s)-\alpha_{22} \nu_{i n v}^{0}(s)^{2} \partial_{s s} u_{2} N^{0}(s) \partial_{s} u_{3}
\end{aligned}
$$




$$
i_{31}^{(2)}=0
$$

$$
\begin{aligned}
i_{32}^{(2)} & =-\nu_{i n v}^{0}(s)^{3} \partial_{s s} u_{2} \partial_{t t s} u_{2} / \alpha_{12}-\nu_{i n v}^{0}(s)^{3} \partial_{s} u_{2} \partial_{t t s s} u_{2} / \alpha_{12}-3 \nu_{i n v}^{0}(s)^{2} \partial_{s} u_{2} \partial_{t t s} u_{2} \nu_{i n v}^{0}{ }^{\prime}(s) / \alpha_{12} \quad(53) \\
n_{3}^{(2)} & =-\alpha_{22} \nu_{i n v}^{0}(s)^{2} \partial_{s} u_{2} \partial_{s s} u_{2}+\alpha_{22} \nu_{i n v}^{0}(s)^{3} \partial_{s} u_{2} \partial_{s s s s} u_{2} / \alpha_{12}+\alpha_{22} \nu_{i n v}^{0}(s)^{2} \partial_{s} u_{2}{ }^{2} \nu_{i n v}^{0}{ }^{\prime \prime \prime}(s) / \alpha_{12} \\
& -2 \alpha_{22} \nu_{i n v}^{0}(s)^{2} \partial_{s s} u_{2} N^{0}(s) \partial_{s} u_{2}+\alpha_{22} \nu_{i n v}^{0}(s)^{3} \partial_{s s s} u_{2} \partial_{s s} u_{2} / \alpha_{12}+2 \alpha_{22} \nu_{i n v}^{0}(s)^{2} \partial_{s s} u_{2}{ }^{2} \nu_{i n v}^{0}(s) / \alpha_{12} \\
& +\alpha_{22} \nu_{i n v}^{0}(s) \partial_{s} u_{2}{ }^{2}+2 \alpha_{22} \nu_{i n v}^{0}(s) \nu_{i n v}^{0}{ }^{\prime \prime}(s) \nu_{i n v}^{0}{ }^{\prime}(s) \partial_{s} u_{2}{ }^{2} / \alpha_{12}+4 \alpha_{22} \nu_{i n v}^{0}(s) \partial_{s s} u_{2} \nu_{i n v}^{0}{ }^{\prime}(s)^{2} \partial_{s} u_{2} / \alpha_{12} \\
& +2 \alpha_{22} \nu_{i n v}^{0}(s) \partial_{s} u_{2} \partial_{s s} u_{2}-\alpha_{22} \nu_{i n v}^{0}(s) \partial_{s} u_{2}{ }^{2} \nu_{i n v}^{0}(s)-\alpha_{22} \nu_{i n v}^{0}(s)^{2} \partial_{s} u_{2}{ }^{2} N^{0 \prime}(s) \\
& +4 \alpha_{22} \nu_{i n v}^{0}(s)^{2} \partial_{s} u_{2} \partial_{s s} u_{2} \nu_{i n v}^{0}{ }^{\prime \prime}(s) / \alpha_{12}+5 \alpha_{22} \nu_{i n v}^{0}(s)^{2} \partial_{s} u_{2} \partial_{s s s} u_{2} \nu_{i n v}^{0}(s) / \alpha_{12} \\
& -2 \alpha_{22} \nu_{i n v}^{0}(s) \partial_{s} u_{2}{ }^{2} N^{0}(s) \nu_{i n v}^{0}{ }^{\prime}(s)
\end{aligned}
$$

$$
\begin{aligned}
& n_{B C 21}^{(2)}=2 \alpha_{22} \partial_{s} u_{2} \nu_{i n v}^{0}{ }^{\prime 2} \partial_{s} u_{3} / \alpha_{12}+4 \alpha_{22} \partial_{s} u_{2} \partial_{s s} u_{3} \nu_{i n v}^{0}{ }^{\prime} / \alpha_{12}+2 \alpha_{22} \partial_{s} u_{2} \partial_{s} u_{3} \nu_{i n v}^{0}{ }^{\prime \prime} / \alpha_{12} \\
& +4 \alpha_{22} \partial_{s s} u_{2} \partial_{s} u_{3} \nu_{i n v}^{0}{ }^{\prime} / \alpha_{12}+2 \alpha_{22} \partial_{s s} u_{2} \partial_{s s} u_{3} / \alpha_{12}+\alpha_{22} \partial_{s} u_{2} \partial_{s s s} u_{3} / \alpha_{12}+\alpha_{22} \partial_{s s s} u_{2} \partial_{s} u_{3} / \alpha_{12} \\
& i_{B C 21,1}^{(2)}=-2 \partial_{t s} u_{2} \partial_{t s} u_{3} / \alpha_{12}, \quad i_{B C 21,2}^{(2)}=-\partial_{s} u_{2} \partial_{t t s} u_{3} / \alpha_{12}-\partial_{t t s} u_{2} \partial_{s} u_{3} / \alpha_{12} \\
& n_{B C 22}^{(2)}=2 \alpha_{22} \partial_{s} u_{2} \partial_{s} u_{3} \nu_{i n v}^{0}{ }^{\prime} / \alpha_{12}+\alpha_{22} \partial_{s s} u_{2} \partial_{s} u_{3} / \alpha_{12}+\alpha_{22} \partial_{s} u_{2} \partial_{s s} u_{3} / \alpha_{12} \\
& n_{B C 3}^{(2)}=(1 / 2) \alpha_{22} \partial_{s} u_{2}^{2}
\end{aligned}
$$

\section{Appendix III: Parameters employed in the secular terms and the Galerkin discretization}

$$
\begin{aligned}
& \delta_{k, k}=\int_{0}^{1}\left[\psi_{2, k} \psi_{2, k}+\psi_{3, k} \psi_{3, k}\right] \mathrm{d} s, \quad \xi_{1}=\int_{0}^{1}\left[\psi_{2, k}^{2}\right] \mathrm{d} s \\
& \xi_{2}=\int_{0}^{1}\left[\psi_{2, k} \nu_{i n v}^{0}\left(\nu_{i n v}^{0}\right)\left(\psi_{2, k}^{\prime}\right)\right] \mathrm{d} s+\frac{1}{2}\left(\int_{0}^{1}\left[\psi_{2, k} \nu_{i n v}^{0}{ }^{2}\left(\psi_{2, k}^{\prime \prime}\right)\right] \mathrm{d} s\right), \quad \xi_{3}=\int_{0}^{1}\left[\psi_{2, k} \psi_{3, k}\right] \mathrm{d} s \\
& \xi_{4}=\int_{0}^{1}\left[\psi_{3, k}^{2}\right] \mathrm{d} s, \xi_{5}=\int_{0}^{1} \psi_{2, k} \psi_{2, k}^{\prime \prime} \nu_{i n v}^{0}{ }^{2} \mathrm{~d} s, \xi_{6}=\int_{0}^{1} \psi_{2, k} \nu_{i n v}^{0}{ }^{\prime} \nu_{i n v}^{0} \psi_{2, k}^{\prime} \mathrm{d} s, \xi_{7}=\int_{0}^{1} \psi_{2, k} \nu_{i n v}^{0}{ }^{\prime} \nu_{i n v}^{0}{ }^{\prime \prime} \psi_{2, k}^{\prime} \mathrm{d} s \\
& \xi_{8}=\int_{0}^{1} \psi_{2, k} \nu_{i n v}^{0}{ }^{\prime 2} \psi_{2, k}^{\prime \prime} \mathrm{d} s, \xi_{9}=\int_{0}^{1} \psi_{2, k} \nu_{i n v}^{0}{ }^{\prime} \nu_{i n v}^{0} \psi_{2, k}^{\prime \prime \prime} \mathrm{d} s, \xi_{10}=\int_{0}^{1} \psi_{2, k} \nu_{i n v}^{0} \nu_{i n v}^{0}{ }^{\prime \prime \prime} \psi_{2, k}^{\prime} \mathrm{d} s \\
& \xi_{11}=\int_{0}^{1} \psi_{2, k} \nu_{i n v}^{0} \nu_{i n v}^{0}{ }^{\prime \prime} \psi_{2, k}^{\prime \prime} \mathrm{d} s, \xi_{12}=\int_{0}^{1} \psi_{2, k} \nu_{i n v}^{0}{ }^{2} \psi_{2, k}^{(I V)} \mathrm{d} s, \xi_{13}=\int_{0}^{1} \psi_{2, k} \nu_{i n v}^{0}{ }^{\prime} \psi_{2, k}^{\prime} \mathrm{d} s \\
& \xi_{14}=\int_{0}^{1} \psi_{2, k} \psi_{2, k}^{\prime \prime} \mathrm{d} s, \xi_{15}=\int_{0}^{1} \psi_{2, k} \psi_{2, k}^{\prime \prime} \nu_{i n v}^{0} \mathrm{~d} s, \xi_{16}=\int_{0}^{1} \psi_{3, k} \psi_{3, k}^{\prime \prime} \mathrm{d} s .
\end{aligned}
$$




$$
\begin{aligned}
& \Gamma_{1, k}=\xi_{33}+\xi_{221}-\frac{1}{2} C_{2} / \alpha_{12}-2 \xi_{222} / \alpha_{12}, \quad \Gamma_{2, k, i}=\Gamma_{2, k, i, \omega_{2, k}^{2}} \omega_{2, k}^{2}+\Gamma_{2, k, i, C o n s} \\
& \Gamma_{2, k, i, \omega_{2, k}^{2}}=-C_{4, \omega_{2, k}^{2}} / \alpha_{12}-5 \chi_{2 d 53} / \alpha_{12}-6 \chi_{2 c 39} / \alpha_{12}-15 \chi_{2 d 46} / \alpha_{12}-5 \chi_{2 d 45} / \alpha_{12}-2 \chi_{2 c 55} / \alpha_{12} \\
& -2 \chi_{2 c 56} / \alpha_{12}-15 \chi_{3 b 56} / \alpha_{12}-5 \chi_{3 b 31} / \alpha_{12}-5 \chi_{3 b 27} / \alpha_{12}
\end{aligned}
$$

$$
\begin{aligned}
& \Gamma_{2, k, R}=\Gamma_{2, k, R, \omega_{2, k}^{2}} \omega_{2, k}^{2}+\Gamma_{2, k, R, \text { Cons }} \\
& \Gamma_{2, k, R, \omega_{2, k}^{2}}=-\frac{1}{2}\left(4 \chi_{2 c 41}+10 \chi_{2 d 50}+4 \chi_{2 d 26}+10 \chi_{2 d 28}+8 \chi_{2 c 47}-40 \chi_{3 a 27}+8 \chi_{2 c 46}+4 \chi_{3 b 71}\right. \\
& +30 \chi_{3 b 33}+4 \chi_{3 b 78}+32 \chi_{2 a 3}-80 \chi_{2 b 3}+30 \chi_{2 d 7}+10 \chi_{3 b 57}+2 C_{3, \omega_{2, k}^{2}}+24 \chi_{2 c 6} \\
& \left.+12 \chi_{2 b 17}-80 \chi_{3 a 7}+10 \chi_{3 b 40}+24 \chi_{2 a 5}+12 \chi_{2 d 5}+4 \chi_{2 c 59}-20 \chi_{2 b 5}+4 \chi_{2 d 12}-20 \chi_{3 a 10}\right) / \alpha_{12}
\end{aligned}
$$

$$
\Gamma_{3, k}=-\frac{1}{2} \xi_{33}-\frac{1}{2} \xi_{221}, \quad \zeta_{2, k}=\zeta_{2} \xi_{221}+\zeta_{3} \xi_{33}
$$

where,

$$
\begin{aligned}
& C_{2}=-\left.2\left(\left.\psi_{2, k}^{\prime}\right|_{s=1}\right) \psi_{2, k}\right|_{s=1}, \quad C_{4, \omega_{2, k}^{2}}=-\left.\left[\left(\left.\psi_{2, k}^{\prime}\right|_{s=1}\right)\left(\left.h_{32, i}^{\prime}\right|_{s=1}\right)+\left(\left.h_{22, i}^{\prime}\right|_{s=1}\right)\left(\left.\psi_{3, k}^{\prime}\right|_{s=1}\right)\right] \psi_{2, k}\right|_{s=1}, \\
& C_{3, \omega_{2, k}^{2}}=-\left[\left(\left.\psi_{2, k}^{\prime}\right|_{s=1}\right)^{3}-3\left(\left.\psi_{2, k}^{\prime}\right|_{s=1}\right)\left(\left.\psi_{3, k}^{\prime}\right|_{s=1}\right)^{2}+\left(\left.\psi_{2, k}^{\prime}\right|_{s=1}\right)\left(\left.h_{32, R}^{\prime}\right|_{s=1}\right)+2\left(\left.\psi_{2, k}^{\prime}\right|_{s=1}\right)\left(\left.h_{31}^{\prime}\right|_{s=1}\right)\right. \\
& \left.+\left(\left.h_{22, R}^{\prime}\right|_{s=1}\right)\left(\left.\psi_{3, k}^{\prime}\right|_{s=1}\right)+2\left(\left.h_{21}^{\prime}\right|_{s=1}\right)\left(\left.\psi_{3, k}^{\prime}\right|_{s=1}\right)\right]\left.\psi_{2, k}\right|_{s=1}, \\
& C_{4, \omega_{2, k}^{2}}=-\left.\left[\left(\left.\psi_{2, k}^{\prime}\right|_{s=1}\right)\left(\left.h_{32, i}^{\prime}\right|_{s=1}\right)+\left(\left.h_{22, i}^{\prime}\right|_{s=1}\right)\left(\left.\psi_{3, k}^{\prime}\right|_{s=1}\right)\right] \psi_{2, k}\right|_{s=1}
\end{aligned}
$$

$\Gamma_{2, k, i, C o n s}, \Gamma_{2, k, R, \text { Cons }}$ and $\chi$ 's are omitted due to the brevity.

$\gamma_{k, i}=\frac{1}{2} \Gamma_{2, k, R} /\left(\omega_{2, k} \Gamma_{1, k}\right), \quad \gamma_{k, R}=-\frac{1}{2} \Gamma_{2, k, i} /\left(\omega_{2, k} \Gamma_{1, k}\right), \quad \gamma_{1, k}=\lambda^{2} \delta \Gamma_{3, k} /\left(\omega_{2, k} \Gamma_{1, k}\right), \quad \gamma_{2, k}=-\zeta_{2, k} \omega_{2, k} / \Gamma_{1, k}$

\section{Appendix V: The employed parameters in the Galerkin discretization}

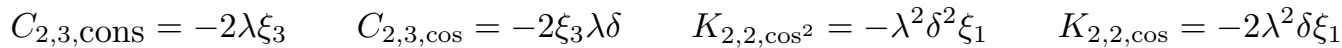

$$
\begin{aligned}
& K_{2,2, \mathrm{cons}}=-\lambda^{2} \xi_{1}+\alpha_{22} \xi_{13}-\alpha_{22} \xi_{14}+\alpha_{22} \xi_{15}+\alpha_{22} \xi_{7} / \alpha_{12}+2 \alpha_{22} \xi_{8} / \alpha_{12}+4 \alpha_{22} \xi_{9} / \alpha_{12} \\
& +\alpha_{22} \xi_{10} / \alpha_{12}+3 \alpha_{22} \xi_{11} / \alpha_{12}+\alpha_{22} \xi_{12} / \alpha_{12}, C_{3,2, \mathrm{cons}}=2 \lambda \xi_{3} \quad C_{3,2, \mathrm{cos}}=2 \xi_{3} \lambda \delta, \\
& K_{3,3, \mathrm{cons}}=-\lambda^{2} \xi_{4}-\alpha_{22} \xi_{16} \quad K_{3,3, \cos ^{2}}=-\lambda^{2} \delta^{2} \xi_{4}, K_{3,3, \mathrm{cos}}=-2 \lambda^{2} \delta \xi_{4},
\end{aligned}
$$




\section{Acknowledgments}

This work has been financially supported by the research deputy of Shahrekord University. The grant number was 97GRN1M1754.

\section{Conflict of interest statement}

The authors declare that they have no known competing financial interests or personal relationships that could have appeared to influence the work reported in this paper.

[1] Arena, A., Lacarbonara, W., Marzocca, P. Nonlinear Aeroelastic Formulation and Postflutter Analysis of Flexible High-Aspect-Ratio Wings, Journal of Aircraft, 50(6):1748-1764 (2013).

[2] Hodges, D. H., Dowell, E. H., Nonlinear equations of motion for the elastic bending and torsion of twisted nonuniform rotor blades, NASA TN D-7818, (1974).

[3] Crespo da Silva, M. R. M., Hodges, D. H., Nonlinear flexure and torsion of rotating beams with application to helicopter rotor blades-I. Formulation, Vertica, 10(2):151-169 (1986).

[4] Crespo da Silva M.R.M., Hodges D.H., Nonlinear flexure and torsion of rotating beams with application to helicopter rotor blades-II. response and stability results, Vertica, 10(2) (1986) 171-186.

[5] Hodges, D.H., A mixed variational formulation based on exact intrinsic equations for dynamics of moving beams, Int. J. Solids Struct., 26(11):1253-1273 (1990).

[6] Hodges, D.H., Comment on "Flexural behavior of a rotating sandwich tapered beam" and on "Dynamic analysis for free vibrations of rotating sandwich tapered beams", AIAA Journal, 33(6):1168-1170 (1995).

[7] Pesheck, E., Pierre, C., Shaw, S. W.: Modal reduction of a nonlinear rotating beam through nonlinear normal modes, J. Vib. Acoust., 124, 229-236 (2002).

[8] Avramov, K. V., Pierre, C., Shyriaieva, N. V., Nonlinear equations of flexural-flexural-torsional oscillations of rotating beams with arbitrary cross-section, Int. Appl. Mech., 44(5):582-589 (2008).

[9] Saravia C.M., Machado S.P., Cortínez V.H., Dynamic stability of rotating thin-walled composite beams, Mec. Comput., XXVIII (2009) 3297-3317.

[10] Turhan Ö., Bulut G., On nonlinear vibrations of a rotating beam, J. Sound. Vib., 322(1-2) (2009) 314-335.

[11] Valverde J., García-Vallejo D., Stability analysis of a substructured model of the rotating beam, Nonlinear. Dyn., $\mathbf{5 5}(4)$ (2009) 355-372.

[12] Qin, Y., Li, Y. H., Influences of hygrothermal environment and installation mode on vibration characteristics of a rotating laminated composite beam, Mech. Syst. Signal. Pr. 91 (2017) 23-40.

[13] Sabater, A. B., Rhoads, J. F., Parametric system identification of resonant micro/nanosystems operating in a nonlinear response regime, Mech. Syst. Signal. Pr., 84 (2017) 241-264.

[14] Tresser, S., Bucher, I., Balancing fast flexible gyroscopic systems at low speed using parametric excitation, Mech. Syst. Signal. Pr. 130 (2019) 452-469.

[15] Lacarbonara W., Arvin H., Bakhtiari-Nejad F., A geometrically exact approach to the overall dynamics of elastic rotating blades - part 1: linear modal properties, Nonlinear. Dyn., 70(1) (2012) 659-675.

[16] Arvin H., Bakhtiari-Nejad F., Lacarbonara W., A geometrically exact approach to the overall dynamics of elastis rotating blades - part 2: nonlinear normal modes in flapping, Nonlinear. Dyn., 70(3) (2012) 2279-2301.

[17] Arvin H., Lacarbonara W., A fully nonlinear dynamic formulation for rotating composite beams: nonlinear normal modes in flapping, Compos. Struct., 109 (2014) 93-105.

[18] Arvin H., Bakhtiari-Nejad F., Nonlinear free vibration analysis of rotating composite Timoshenko beams, Compos. Struct., 96 (2013) 29-43. 
[19] Liu, T., Zhang, W., Mao, J. J., Zheng, Y. . Nonlinear breathing vibrations of eccentric rotating composite laminated circular cylindrical shell subjected to temperature, rotating speed and external excitations, Mech. Syst. Signal. Pr. 127 (2019) 463-498.

[20] Arvin H., On Parametrically Excited Vibration and Stability of Beams with Varying Rotating Speed, Iran J Sci Technol Trans Mech Eng, 43(2) (2019) 177-185.

[21] Arvin, H., Tang, Y. Q., Ahmadi Nadooshan, A., Dynamic stability in principal parametric resonance of rotating beams: Method of multiple scales versus differential quadrature method, Int. J. Non-Linear Mech., 85 (2016) $118-125$.

[22] Heidari M., Arvin H., Nonlinear free vibration analysis of functionally graded rotating composite Timoshenko beams reinforced by carbon nanotubes, J. Vib. Control, 25(14) (2019) 2063-2078.

[23] Arena A., Taló M., Snyder M. P., Lacarbonara W., Enhancing flutter stability in nanocomposite thin panels by harnessing CNT/polymer dissipation, Mech. Res. Commun. 104103495 (2020) 1-6.

[24] Nayfeh, A. H., Mook, D. T., Nonlinear Oscillations, Wiley-Interscience, New York, (1979).

[25] Meirovitch L., Principles and Techniques of Vibrations, first ed., Prentice-Hall, New Jersey, 1997.

[26] Lacarbonara W., Nonlinear Structural Mechanics. Theory, Dynamical Phenomena, and Modeling, first ed., Springer, New York, 2013.

[27] Newmark, N. M. (1959) A method of computation for structural dynamics. J. Eng. Mech., ASCE, 85 (3) 67-94. 